\title{
Wave-activity conservation laws and stability theorems for semi-geostrophic dynamics: part 2. pseudoenergy-based theory
}

Article

Published Version

Kushner, P. J. and Shepherd, T. G. (1995) Wave-activity conservation laws and stability theorems for semi-geostrophic dynamics: part 2. pseudoenergy-based theory. Journal Of Fluid Mechanics, 290. pp. 105-129. ISSN 0022-1120 doi: https://doi.org/10.1017/S0022112095002436 Available at https://centaur.reading.ac.uk/32893/

It is advisable to refer to the publisher's version if you intend to cite from the work. See Guidance on citing.

Published version at: http://dx.doi.org/10.1017/S0022112095002436

To link to this article DOI: http://dx.doi.org/10.1017/S0022112095002436

Publisher: Cambridge University Press

All outputs in CentAUR are protected by Intellectual Property Rights law, including copyright law. Copyright and IPR is retained by the creators or other copyright holders. Terms and conditions for use of this material are defined in the End User Agreement. 


\section{CentAUR}

Central Archive at the University of Reading

Reading's research outputs online 


\title{
Wave-activity conservation laws and stability theorems for semi-geostrophic dynamics. Part 2. Pseudoenergy-based theory
}

\author{
By PAUL J. KUSHNER AND THEODORE G. SHEPHERD \\ Department of Physics, University of Toronto, Toronto, Canada M5S 1A7
}

(Received 31 May 1994 and in revised form 12 December 1994)

This paper represents the second part of a study of semi-geostrophic (SG) geophysical fluid dynamics. SG dynamics shares certain attractive properties with the better known and more widely used quasi-geostrophic (QG) model, but is also a good prototype for balanced models that are more accurate than QG dynamics. The development of such balanced models is an area of great current interest. The goal of the present work is to extend a central body of QG theory, concerning the evolution of disturbances to prescribed basic states, to SG dynamics. Part 1 was based on the pseudomomentum; Part 2 is based on the pseudoenergy.

A pseudoenergy invariant is a conserved quantity, of second order in disturbance amplitude relative to a prescribed steady basic state, which is related to the time symmetry of the system. We derive such an invariant for the semi-geostrophic equations, and use it to obtain: (i) a linear stability theorem analogous to Arnol'd's 'first theorem'; and (ii) a small-amplitude local conservation law for the invariant, obeying the group-velocity property in the WKB limit. The results are analogous to their quasi-geostrophic forms, and reduce to those forms in the limit of small Rossby number.

The results are derived for both the $f$-plane Boussinesq form of semi-geostrophic dynamics, and its extension to $\beta$-plane compressible flow by Magnusdottir \& Schubert. Novel features particular to semi-geostrophic dynamics include apparently unnoticed lateral boundary stability criteria. Unlike the boundary stability criteria found in the first part of this study, however, these boundary criteria do not necessarily preclude the construction of provably stable basic states.

The interior semi-geostrophic dynamics has an underlying Hamiltonian structure, which guarantees that symmetries in the system correspond naturally to the system's invariants. This is an important motivation for the theoretical approach used in this study. The connection between symmetries and conservation laws is made explicit using Noether's theorem applied to the Eulerian form of the Hamiltonian description of the interior dynamics.

\section{Introduction}

Semi-geostrophic (SG) dynamics, first expressed in its three-dimensional form by Hoskins (1975), is a model of fundamental interest and central importance in geophysical fluid dynamics. Next to quasi-geostrophic (QG) dynamics, SG dynamics has been the most widely used and extensively studied 'balanced' model. (Balanced models are reduced models of the primitive equations that describe only the slow-time dynamics. Motions whose characteristic timescales are faster than or equal to the local 
Coriolis period are thus eliminated (see, e.g. Warn et al. 1995).) The SG and QG models share important characteristics. Like the full primitive equations, the inviscid adiabatic forms of both models globally conserve the energy and materially conserve the potential vorticity (PV) and the entropy. In addition, both models have the classical structure of balanced models, namely a single prognostic equation for the potential vorticity, together with an 'invertibility' or 'balance' relation that determines the advecting velocity from the PV (see, e.g. Hoskins, McIntyre \& Robertson 1985).

Despite the family resemblance between the SG and QG models, SG dynamics has distinguishing features that make it a good prototype for accurate higher-order balanced models. First, the scaling assumptions of SG dynamics are far less restrictive than the scaling assumptions of QG dynamics. As a result SG dynamics captures important phenomena, such as frontogenesis, that are not exhibited by QG dynamics (Hoskins 1982). Secondly, like the Charney balance relation, the invertibility relation in SG dynamics is nonlinear. Finally, the SG equations are expressed in their simplest form under a nonlinear coordinate transformation that depends on the dynamical fields. This feature could well extend to other higher-order balanced models as they are developed (see, e.g. Schubert \& Magnusdottir 1994). Therefore, the insight gained by understanding the implications of nonlinear invertibility relations and nonlinear coordinate transformations should apply more widely than to SG dynamics alone.

Because we view SG dynamics as a prototype for higher-order balance models, we are motivated to see to what extent theoretical results based on the simpler QG dynamics can be extended to SG dynamics. We have been focusing in this two-part study on a body of QG theory concerning the evolution of disturbances to prescribed basic states. In the first part of this study (Kushner \& Shepherd 1995, hereinafter referred to as Part 1), we show how some of this QG theory, consisting of wave-activity conservation laws and stability theorems for disturbances to zonal basic states, can be systematically generalized to SG dynamics. In particular, we derive an SG pseudomomentum invariant for disturbances to prescribed parallel basic states, and use it to obtain linear and nonlinear Charney-Stern stability theorems, a local waveactivity flux law, and a theory of wave-zonal-mean-flow interaction.

In this second part we continue this theoretical development by deriving SG waveactivity conservation laws and stability theorems for disturbances to prescribed steady (and in general non-parallel) basic states. The approach is similar to that of Part 1. The pseudoenergy - i.e. the wave-activity invariant for disturbances to steady basic states that is related to the time symmetry of the dynamics - is derived from a variational principle. For steady basic states, the invariant is the sum of the energy and a so-called 'Casimir' functional of the PV and potential-temperature fields. By examining the sign of the invariant for disturbances of arbitrary form, we obtain a stability theorem analogous to Arnol'd's (1966) 'first theorem' for two-dimensional Euler flow and its extension to three-dimensional QG stratified flow (Swaters 1986; McIntyre \& Shepherd 1987, hereinafter referred to as MS87). A local form of the wave-activity conservation law is then developed, and the group-velocity property verified for the particular form of the flux.

One important difference between the results of this paper and the results of Part 1 and MS 87 is the restriction of the stability theorem and of the local wave-activity conservation law to small-amplitude disturbances. This is an unsatisfactory aspect of the SG pseudoenergy analysis. The QG and SG pseudomomenta, and the QG pseudoenergy (MS87; Shepherd 1989; Part 1), are sign-definite for arbitrary finiteamplitude disturbances if the basic states have certain monotonicity properties. This sign-definiteness makes it possible to derive finite-amplitude Liapunov stability 
theorems based on the invariants. The SG pseudoenergy invariant, by contrast, appears not to be sign-definite at finite amplitude. This can be ascribed to the nonquadratic form of the energy in isentropic geostrophic coordinates (IGC) as well as to boundary variability. The non-quadratic form of the energy also accounts for the restriction of the local conservation law to small-amplitude disturbances.

There is also a significant difference between the role of lateral boundaries in the stability theory of this paper and that of Part 1 . In Part 1, it is found that the simple presence of opposing meridional boundaries prevents the construction of basic states that are provably stable by the SG Charney-Stern stability theorem. On the other hand, the presence of opposing lateral boundaries does not necessarily violate the criteria of the SG Arnol'd stability theorem found here. Instead, the criteria depend in a more complex way on the basic flow.

This paper must be read together with certain sections of Part 1 . The $f$-plane Boussinesq system of Hoskins (1975) is introduced and transformed to IGC in Part 1, $\S 2$. The conservation laws for the system are derived in Part $1, \S 4$. In $\S 2$ of this paper, the pseudoenergy is derived from a variational principle, after a discussion of the nature of the basic state. The invariant is shown to reduce, in the small-amplitude small-Rossby-number limit, to the small-amplitude form of the QG pseudoenergy. In $\S 3$, the SG generalization of Arnol'd's first stability theorem that follows from the small-amplitude conservation law of $\$ 2$ is discussed. In $\$ 4$, a local form of the pseudoenergy conservation law is developed.

Magnusdottir \& Schubert's (1990, hereinafter referred to as MSc90; 1991) recent extensions of the SG equations to $\beta$-plane and hemispheric compressible flow represent important advances in the effort to have balanced models realistically represent the atmospheric general circulation over large horizontal and vertical scales. The $\beta$-plane system of MSc90 is discussed in Part $1, \S 9.2$, and its conservation properties described in Part $1, \S 9.4$. The pseudoenergy-based results of $\$ \$ 2-4$ are extended to the MSc90 system in $\$ 5$. The finite-amplitude pseudoenergy for White's (1977) modified QG system is recorded in Appendix B. At small amplitude, this modified-QG pseudoenergy invariant corresponds to the small-Rossby-number form of the $\beta$-plane compressible SG pseudoenergy invariant.

As stated in Part 1, both QG and SG dynamics have an underlying Hamiltonian structure which guarantees sufficient conservation laws for the construction of the wave-activity invariants (see e.g. Shepherd 1990). In $\S 6$, the Hamiltonian structure of the interior dynamics for the $f$-plane Boussinesq (Roulstone \& Norbury 1994) and $\beta$ plane compressible systems (Kushner 1993) is presented. The zonal impulse, Casimir and energy invariants are then all shown to arise naturally from the Hamiltonian structure using Noether's theorem.

The results are summarized and discussed in $\$ 7$.

\section{Pseudoenergy}

A 'wave-activity' invariant is defined to be a conserved quantity that is second order in the amplitude of a disturbance to a prescribed reference or 'basic' state having some continuous symmetry. The 'pseudoenergy' is the wave activity, defined with respect to steady basic states, that is associated with the time symmetry of the system; it is an exact invariant of the nonlinear dynamics.

In this section we construct the pseudoenergy for the $f$-plane Boussinesq form of $\mathrm{SG}$ dynamics. The model is described in Part $1, \S 2$, and its conservation properties are described in Part 1, $\$ 4$. 


\subsection{Basic state}

Consider a steady basic state in physical space. Since

$$
\partial_{t}=X_{t} \partial_{X}+Y_{t} \partial_{Y}+Z_{t} \partial_{Z}+\partial_{T}
$$

$\partial_{t}=0$ implies $\partial_{T}=0$ in the basic state. We use this to prove the following:

Proposition. In the steady basic state: (i) $\sigma$-and $\Psi$-surfaces coincide along each $Z$ surface in the interior, and (ii) $\Psi$ - and Z-surfaces coincide at a material boundary for sign-definite $\sigma$.

Proof. The proof of (i) follows from Part 1 (2.18a) and (2.19) with $\partial_{T}=0$, whence

$$
\partial_{X Y}(\sigma, \Psi)=0, \quad \text { basic state. }
$$

To prove (ii), we write the 'no normal flow' boundary conditions as

$$
\frac{\mathrm{D} x}{\mathrm{D} t} \cdot \hat{n}=(u, v, w) \cdot \hat{n}=0 \text { at material boundaries, }
$$

where $\hat{\boldsymbol{n}}=\left(n_{x}, n_{y}, n_{z}\right)$ is a unit vector normal to the boundaries in physical space. Using Part 1 (2.19) with $\partial_{T}=0$ and Part 1 (2.20), we find

$$
\partial_{X Y}(\Psi, x) \cdot \hat{n}=\sigma \partial_{x y z}(\Psi, x, Z) \cdot \hat{n}=\sigma\left(\nabla_{3} Z \times \nabla_{3} \Psi\right) \cdot \hat{n}=0,
$$

where $\boldsymbol{\nabla}_{3}$ is the three-dimensional gradient in physical coordinates. Part (ii) of the Proposition follows.

Remark. It follows from the Proposition that if the $\sigma-, \Psi$ - and $Z$-fields are continuous throughout the domain, then the $\sigma$-surfaces also coincide with the $\Psi$-and $Z$-surfaces at the boundary. Thus, $P V$ and $\Psi$ are constant at each potentialtemperature surface along the boundary in the steady basic state.

Equation (2.1) allows us to follow the approach of MS87 and define an inverse map $\tilde{\Psi}_{i n t}(\tilde{\sigma}, Z)$ in the interior, in IGC, where here and henceforth tildes indicate basic-state quantities. If $\tilde{\sigma}$ is not monotonic in $\tilde{\Psi}, \tilde{\Psi}_{i n t}$ will be multivalued and additional Lagrangian information will be needed to fully define the inverse map (MS87). At every basic-state surface, we have established through part (ii) of the Proposition and the Remark that there are isentropic distributions $\tilde{\Psi}_{s}(Z)$ and $\tilde{\sigma}_{s}(Z)$, where $s$ is a discrete index specifying the surface. The function $\widetilde{\Psi}_{s}(Z)$, for example, is defined to be the value of $\tilde{\Psi}$ at the $s$-boundary point with isentropic height $Z$. A more complete discussion of these maps may be found in $\S 5.1$ of Part 1 . We note that integrals in IGC over $\tilde{\Psi}_{s}(Z)$ and $\tilde{\Psi}_{i n t}(\sigma, Z)$ are Casimir functionals of the form given in Part 1 (4.11).

\subsection{Finite-amplitude pseudoenergy}

We seek a conserved quantity, $\mathscr{U}$, that is second-order relative to the steady basic state. This requires that $\mathscr{U}$ be of the form

$$
\mathscr{U}=\mathscr{A}[\sigma]-\mathscr{A}[\tilde{\sigma}]
$$

where $\sigma$ and $\tilde{\sigma}$ symbolize all perturbed and basic-state fields, and that $\mathscr{A}$ be extremal at the basic state, i.e.

$$
\delta \mathscr{A}=0 \text { at the basic state. }
$$

As in Part 1, in order to apply a variational principle of the form (2.5) we must include the variation at the boundaries explicitly. Consider an integral $\mathscr{F}$ of a density 
$F$ whose local variation is denoted $\delta F$. Rather than specify limits of integration, we will denote the basic-state region as $\tilde{D}$ and variations to it by $D^{\prime}$. We find for the box domain that

$$
\delta \mathscr{F}=\int_{\tilde{D}} \delta F \mathrm{~d} X \mathrm{~d} Y \mathrm{~d} Z+\left.\sum_{i=1}^{3}\left[\left.\int_{x_{i}, \tilde{D}} F \delta X_{i}\right|_{x_{i}} \mathrm{~d} X_{j} \mathrm{~d} X_{k}\right]\right|_{x_{i}=x_{i 1}} ^{x_{i}=x_{i 2}},
$$

where here and below $\left\{x_{i}\right\}=\{x, y, z\},\left\{X_{i}\right\}=\{X, Y, Z\}$, and the indices $\{i, j, k\}$ are cyclic permutations of $\{1,2,3\}$. In (2.6) we have chosen to parametrize the variations of the limits of IGC integration at the $x$-, $y$-and $z$-surfaces in terms of variations in $X, Y$, and $Z$ respectively. The advantage of this approach is that the displacements are unambiguously defined as long as

$$
0<\left|\frac{\partial x_{i}}{\partial X_{i}}\right|_{X_{j}, X_{k}} \mid<\infty \quad \text { at } x_{i} \text { boundaries, } i=1,2,3 \text {, }
$$

for both perturbed and unperturbed boundaries. We take the gradients to be positive, requiring, in essence, that $(X, Y, Z)$ act as sensible zonal, meridional and vertical coordinates in a statically stable flow with Rossby number less than unity (see remark (iii) in $\S 3$ of Part 1 ).

We note that (2.6) neglects as second-order quantities the variations in the limits of integration along the edges $\left\{\left(x, y_{1}, z_{1}\right): x_{1} \leqslant x \leqslant x_{2}\right\}$, etc., and as third-order quantities the variations at the corners. Hence, to leading order, the limits of integration on all the integrals are taken to be those of the basic state and there is no additional need to consider the variations $D^{\prime}$. The subscripts $\tilde{D}$ on the surface integrals indicate this approximation.

We now consider functional variations on the energy invariant $\mathscr{E}$ (Part 1 (4.2)-(4.3)) and the Casimir invariants $\mathscr{C}$ (Part 1 (4.8), (4.11)). The energy density in physical coordinates is

$$
E=\frac{1}{2}\left(u_{g}^{2}+v_{g}^{2}\right)-f^{2} Z z=f^{2}\left(\frac{1}{2}\left[(x-X)^{2}+(y-Y)^{2}\right]-Z z\right)=\Psi-\phi
$$

(Hoskins 1975; Part 1 (4.2)). The second equality in (2.8) follows from Part 1 (2.9), while the third follows from Part 1 (2.10). As in Part 1, the variational calculations below use the IGC form of the fields. The energy density in IGC is $E^{*}$, defined by

$$
\mathscr{E}=\int E \mathrm{~d} x \mathrm{~d} y \mathrm{~d} z=\int E^{*} \mathrm{~d} X \mathrm{~d} Y \mathrm{~d} Z,
$$

where

$$
E^{*}=(\Psi-\phi) \partial_{X Y Z}(x, y, z)=(\Psi-\phi) \sigma,
$$

after using the final component of Part 1 (2.20).

In Appendix A, §A.1, it is shown that starting with (2.6) and using (2.8)-(2.10) and the invertibility relations Part 1 (2.20), the first variation on the energy functional is given by

$$
\delta \mathscr{E}=\int_{\tilde{D}} \Psi \delta \sigma \mathrm{d} X \mathrm{~d} Y \mathrm{~d} Z+\left.\sum_{i=1}^{3}\left[\left.\int_{x_{i}, \tilde{D}} \Psi \sigma \delta X_{i}\right|_{x_{i}} \mathrm{~d} X_{j} \mathrm{~d} X_{k}\right]\right|_{x_{i}=x_{i 1}} ^{x_{i}-x_{t 2}}
$$

Clearly the energy is not extremal for this basic state as its first variation (2.11) is, in general, non-zero. In the usual way, we define the invariant

$$
\mathscr{A}=\mathscr{E}+\mathscr{C},
$$

whose conservation is guaranteed by Part 1 (4.3) and (4.8), and then determine $\mathscr{C}$ such that $\mathscr{A}$ satisfies (2.5). 
For any Casimir $\mathscr{C}$ of the form Part 1 (4.1)

$$
\delta \mathscr{C}=\left.\int_{\tilde{D}} \frac{\partial C^{*}}{\partial \sigma}\right|_{Z} \delta \sigma \mathrm{d} X \mathrm{~d} Y \mathrm{~d} Z+\left.\sum_{i=1}^{3}\left[\left.\int_{x_{i}, \tilde{D}} C^{*} \delta X_{i}\right|_{x_{i}} \mathrm{~d} X_{j} \mathrm{~d} X_{k}\right]\right|_{x_{i}-x_{i 1}} ^{x_{i}=x_{i 2}} .
$$

In order to satisfy (2.5), we require from (2.11) and (2.13) that

$$
\left.\begin{array}{rl}
\left.\frac{\partial C^{*}}{\partial \sigma}\right|_{Z} & =-\Psi \quad \text { in the interior, basic state, } \\
C^{*} & =-\Psi \sigma \quad \text { at all boundaries, basic state. }
\end{array}\right\}
$$

A Casimir density satisfying (2.14) takes the local form

$$
C^{*}(\sigma, Z)=-\int_{\tilde{\sigma}_{s}(Z)}^{\sigma} \tilde{\Psi}_{i n t}(\hat{\sigma}, Z) \mathrm{d} \hat{\sigma}-\tilde{\sigma}_{s}(Z) \tilde{\Psi}_{s}(Z) .
$$

We now consider a finite-amplitude disturbance to the basic state. We define disturbance quantities $\Psi^{\prime}$ and $\sigma^{\prime}$ by

$$
\Psi=\tilde{\Psi}+\Psi^{\prime}, \quad \sigma=\tilde{\sigma}+\sigma^{\prime}
$$

where we have dropped the $\delta$-variational notation to emphasize that the disturbance can be large. We leave the limits of integration of both the $\tilde{D}$ and the $D^{\prime}$ regions unspecified for the moment.

The inverse maps $\tilde{\Psi}_{i n t}(\sigma, Z), \tilde{\Psi}_{s}(Z)$ and $\tilde{\sigma}_{s}(Z)$ are Casimir functions defined over the range of values of $\sigma$ and $Z$ present in the basic state. If the disturbance (2.16) introduces values outside that range, then these functions must be extended to include such values. One can make this extension arbitrarily without compromising the fact that $C^{*}$ is a Casimir density, and is therefore conserved (cf. Arnol'd 1966). With this possibility in mind, using (2.15) we obtain

$$
\begin{aligned}
\mathscr{U}=\int_{\tilde{D}}\left(E^{*}-\tilde{E}^{*}\right) \mathrm{d} X \mathrm{~d} Y \mathrm{~d} Z-\int_{\tilde{D}+D^{\prime}}\left[\int_{\tilde{\sigma}}^{\sigma} \tilde{\Psi}_{i n t}(\hat{\sigma}, Z) \mathrm{d} \hat{\sigma}\right] \mathrm{d} X \mathrm{~d} Y \mathrm{~d} Z \\
+\int_{D^{\prime}}\left[E^{*}-\tilde{\Psi}_{i n t}(\tilde{\sigma}, Z) \tilde{\sigma}+\int_{\tilde{\sigma}_{s}(Z)}^{\bar{\sigma}} \hat{\sigma} \frac{\partial \tilde{\Psi}_{i n t}}{\partial \hat{\sigma}} \mathrm{d} \hat{\sigma}\right] \mathrm{d} X \mathrm{~d} Y \mathrm{~d} Z .
\end{aligned}
$$

In deriving (2.17) we have used the identity

$$
\tilde{\Psi}_{i n t}\left(\tilde{\sigma}_{s}(Z), Z\right)=\tilde{\Psi}_{s}(Z)
$$

The expression for $\mathscr{U}$ defines the finite-amplitude pseudoenergy in terms of both surface and interior contributions.

\subsection{Comparison with $Q G$ theory and small-amplitude reduction}

We now show that the small-amplitude form of the pseudoenergy invariant reduces to the small-amplitude form of the QG invariant in the limit of small Rossby number. We will also see in $\$ 3$ that the conservation law for the linearized invariant directly yields a stability theorem for perturbations to steady states, analogous to the small-amplitude versions of Arnol'd's (1966) first stability theorem and its extension to QG stratified flow (Swaters 1986; MS87).

In Appendix A, $\S \mathrm{A} .1$, it is shown that the small-amplitude form of the interior contribution to the pseudoenergy is

$$
\mathscr{U}_{i n t}=\int_{\tilde{D}} \frac{1}{2}\left[f^{2} a_{l m} x_{l}^{\prime} \dot{x}_{m}^{\prime}-\tilde{\Psi}_{\tilde{\sigma}}\left(\sigma^{\prime}\right)^{2}\right] \mathrm{d} X \mathrm{~d} Y \mathrm{~d} Z
$$


where $\tilde{\Psi}_{\tilde{\sigma}}$ is the basic-state partial derivative of $\tilde{\Psi}=\tilde{\Psi}_{i n t}$ with respect to $\tilde{\sigma}$, summation over the indices $l, m$ is implicit, and the matrix of coefficients in the quadratic form is

$$
a_{l m}=\left(\begin{array}{lll}
\partial_{Y Z}(\tilde{y}, \tilde{z}) & \partial_{Z X}(\tilde{y}, \tilde{z}) & \partial_{X Y}(\tilde{y}, \tilde{z}) \\
\partial_{Y Z}(\tilde{z}, \tilde{x}) & \partial_{Z X}(\tilde{z}, \tilde{x}) & \partial_{X Y}(\tilde{z}, \tilde{x}) \\
\partial_{Y Z}(\tilde{x}, \tilde{y}) & \partial_{Z X}(\tilde{x}, \tilde{y}) & \partial_{X Y}(\tilde{x}, \tilde{y})
\end{array}\right) .
$$

It is convenient to rewrite (2.19) in the form

$$
a_{l m}=\tilde{\sigma}\left(\begin{array}{ccc}
X_{\tilde{x}} & Y_{\tilde{x}} & Z_{\tilde{x}} \\
X_{\tilde{y}} & Y_{\tilde{y}} & Z_{\tilde{y}} \\
X_{\tilde{z}} & Y_{\tilde{z}} & Z_{\bar{z}}
\end{array}\right) .
$$

The matrix $a_{l m}$ is symmetric according to generalized thermal-wind relations that may be derived from Part 1 (2.20). Cullen \& Purser (1984) and Shutts \& Cullen (1987) discuss various ways in which this matrix arises in SG theory. Most importantly, the matrix is positive definite when the basic flow is statically and inertially stable; such conditions are required for SG dynamics to be meaningful, since if they were violated then unbalanced flow would likely ensue. We see this as follows: the matrix $a_{l m}$ is positive definite if

$$
a_{33}>0, \quad \operatorname{det}\left(\begin{array}{ll}
a_{22} & a_{23} \\
a_{32} & a_{33}
\end{array}\right)>0, \quad \operatorname{det} a_{l m}>0 .
$$

In the present case these conditions reduce to

$$
\tilde{\sigma} Z_{\bar{z}}>0, \quad \tilde{\sigma} \tilde{x}_{X}>0, \quad \tilde{\sigma}^{2}>0 .
$$

The final condition is trivial; for $\tilde{\sigma}>0$, the first and second conditions correspond respectively to static stability and inertial stability.

In the absence of boundary variability, $\mathscr{U}_{i n t}$ is conserved. Without this restriction, a conservation law including boundary variations may be derived directly from the equations of motion as follows. We define the linearized disturbance $\sigma^{\prime}$ from Part 1 (2.20) by

$$
\sigma^{\prime}=\sum_{i=1}^{3} \partial_{X Y Z}\left(x_{i}^{\prime}, \tilde{x}_{j}, \tilde{x}_{k}\right)
$$

where $\{i, j, k\}$ are cyclic permutations of $\{1,2,3\}$. From Part 1 (2.20) we also obtain

$$
\Psi_{X_{i}}^{\prime}=-f^{2} x_{i}^{\prime}
$$

Using (2.19) and (2.23)-(2.24), we find

$$
\Psi^{\prime} \sigma_{T}^{\prime}=\frac{\partial}{\partial T}\left(\frac{1}{2} f^{2} a_{l m} x_{l}^{\prime} x_{m}^{\prime}\right)+\sum_{i=1}^{3} \partial_{X Y Z}\left(\Psi^{\prime}\left[x_{i}^{\prime}\right]_{T}, \tilde{x}_{j}, \tilde{x}_{k}\right)
$$

Taking the time derivative of $\mathscr{U}_{i n t}$ and using the linearization of Part $1(2.18 a-d)$ then yields

$$
\begin{aligned}
\frac{\mathrm{d} \mathscr{U}_{i n t}}{\mathrm{~d} t} & =\int_{\tilde{D}}\left[\left(\Psi^{\prime}-\tilde{\Psi}_{\tilde{\sigma}} \sigma^{\prime}\right) \sigma_{T}^{\prime}-\sum_{i=1}^{3} \partial_{X Y Z}\left(\Psi^{\prime}\left[x_{i}^{\prime}\right]_{T}, \tilde{x}_{j}, \tilde{x}_{k}\right)\right] \mathrm{d} X \mathrm{~d} Y \mathrm{~d} Z \\
& =\int_{\tilde{D}} \partial_{X Y}\left(\tilde{\sigma}, \frac{1}{2 f}\left(\Psi^{\prime}-\tilde{\Psi}_{\tilde{\sigma}} \sigma^{\prime}\right)^{2}\right) \mathrm{d} X \mathrm{~d} Y \mathrm{~d} Z-\left.\sum_{i=1}^{3}\left[\int_{x_{i}} \Psi^{\prime}\left[x_{i}^{\prime}\right]_{T} \mathrm{~d} \tilde{x}_{j} \mathrm{~d} \tilde{x}_{k}\right]\right|_{x_{i}=x_{i 1}} ^{x_{i}-x_{i 2}}
\end{aligned}
$$




$$
=\left.\sum_{i=1}^{3}\left[\int_{x_{i}} \frac{1}{f} \Psi^{\prime}\left[\partial_{X Y}\left(\tilde{\Psi}, x_{i}^{\prime}\right)+\partial_{X Y}\left(\Psi^{\prime}, \tilde{x}_{i}\right)\right] \mathrm{d} \tilde{x}_{j} \mathrm{~d} \tilde{x}_{k}\right]\right|_{x_{i}-x_{i 1}} ^{x_{i}-x_{i q}}
$$

In (2.26) we have also used the identity

$$
\partial_{X Y}\left(\tilde{\sigma}, \tilde{\Psi}_{\tilde{\sigma}}\right)=0 \text { in the interior, basic state, }
$$

which follows from (2.1). We have changed variables to obtain the boundary terms in $(2.26 b)$. The volume integral over the horizontal Jacobian in $(2.26 b)$ vanishes since, by the Proposition, $\tilde{\sigma}$ is constant along the boundary for fixed $Z$.

Recalling (2.3), we define at each surface the basic-state functions

$$
\frac{\nabla \tilde{\Psi}}{\nabla \tilde{x}_{i}}=\frac{\tilde{\Psi}_{X}}{\left(\tilde{x}_{i}\right)_{X}}=\frac{\tilde{\Psi}_{Y}}{\left(\tilde{x}_{i}\right)_{Y}} \text { at } x_{i} \text { surface, basic state, }
$$

with the property, analogous to (2.27), that

$$
\partial_{X Y}\left(\tilde{x}_{i}, \frac{\nabla \tilde{\Psi}}{\nabla \tilde{x}_{i}}\right)=0 \text { at } x_{i} \text { surface, basic state. }
$$

From the linearized boundary equations Part $1(2.18 b-d)$, and (2.28)-(2.29) above, at the $x_{i}$ boundary

$$
\frac{1}{2} \frac{\nabla \tilde{\Psi} \tilde{x}_{i}}{\partial\left(x_{i}^{\prime}\right)^{2}} \partial=-\partial_{X Y}\left[\tilde{x}_{i}, \frac{1}{2 f}\left(\frac{\nabla \tilde{\Psi}}{\nabla \tilde{x}_{i}}\right)^{2}\left(x_{i}^{\prime}\right)^{2}\right]-\frac{1}{f} x_{i}^{\prime} \partial_{X Y}\left(\Psi^{\prime}, \tilde{\Psi}\right)
$$

Defining the boundary contribution to the pseudoenergy by

$$
\mathscr{U}_{s}=\left.\sum_{i=1}^{3}\left[\int_{x_{i}} \frac{1}{2} \frac{\nabla \tilde{\Psi}}{\nabla \tilde{x}_{i}}\left(x_{i}^{\prime}\right)^{2} \mathrm{~d} \tilde{x}_{j} \mathrm{~d} \tilde{x}_{k}\right]\right|_{x_{i}-x_{i 1}} ^{x_{i}-x_{i 2}}
$$

we obtain from (2.26) and (2.28)-(2.31)

$$
\begin{aligned}
\frac{\mathrm{d}}{\mathrm{d} t}\left(\mathscr{U}_{i n t}+\mathscr{U}_{\delta}\right) & =\left.\sum_{i=1}^{3}\left\{-\int_{x_{i}} \partial_{X Y}\left[\tilde{x}_{i}, \frac{1}{2 f}\left(\Psi^{\prime}-\frac{\nabla \tilde{\Psi}}{\nabla \tilde{x}_{i}} x_{i}^{\prime}\right)^{2}\right] \mathrm{d} \tilde{x}_{j} \mathrm{~d} \tilde{x}_{k}\right\}\right|_{x_{i}-x_{i 1}} ^{x_{i}-x_{i 2}} \\
& =\left.\sum_{i=1}^{3}\left\{-\int_{x_{i}} \tilde{\sigma} \partial_{\tilde{x} \tilde{y} z}\left[\tilde{x}_{i}, \frac{1}{2 f}\left(\Psi^{\prime}-\frac{\nabla \tilde{\Psi}}{\nabla \tilde{x}_{i}} x_{i}^{\prime}\right)^{2}, Z\right] \mathrm{d} \tilde{x}_{j} \mathrm{~d} \tilde{x}_{k}\right\}\right|_{x_{i}-x_{i 1}} ^{x_{i}-x_{i z}} \\
& =\left.\sum_{i=1}^{3}\left\{-\int_{x_{i}} \tilde{\sigma} \partial_{\tilde{x}_{j} \tilde{x}_{k}}\left[\frac{1}{2 f}\left(\Psi^{\prime}-\frac{\nabla \tilde{\Psi}}{\nabla \tilde{x}_{i}} x_{i}^{\prime}\right)^{2}, Z\right] \mathrm{d} \tilde{x}_{j} \mathrm{~d} \tilde{x}_{k}\right\}\right|_{x_{i}-x_{i 1}} ^{x_{i}-x_{i 2}} \\
& =\int_{D}-\partial_{\tilde{x} \tilde{y} \tilde{z}}\left[\tilde{\sigma}, \frac{1}{2 f}\left(\Psi^{\prime}-\frac{\nabla \tilde{\Psi}}{\nabla \tilde{x}_{i}} x_{i}^{\prime}\right)^{2}, Z\right] \mathrm{d} \tilde{x} \mathrm{~d} \tilde{y} \mathrm{~d} \tilde{z} \\
& =\int_{\tilde{D}}-\partial_{X Y}\left[\tilde{\sigma}, \frac{1}{2 f}\left(\Psi^{\prime}-\frac{\nabla \tilde{\Psi}}{\nabla \tilde{x}_{i}} x_{i}^{\prime}\right)^{2}\right] \mathrm{d} X \mathrm{~d} Y \mathrm{~d} Z \\
& =0
\end{aligned}
$$


where we have again used the Proposition. In summary, the conservation law for the small-amplitude pseudoenergy is

$$
\frac{\mathrm{d}}{\mathrm{d} t}\left\{\int_{\tilde{D}} \frac{1}{2}\left[f^{2} a_{l m} x_{l}^{\prime} x_{m}^{\prime}-\tilde{\Psi}_{\tilde{\sigma}}\left(\sigma^{\prime}\right)^{2}\right] \mathrm{d} X \mathrm{~d} Y \mathrm{~d} Z+\left.\sum_{i=1}^{3}\left[\int_{x_{i}} \frac{1}{2} \frac{\nabla \tilde{\Psi}}{\nabla \tilde{x}_{i}}\left(x_{i}^{\prime}\right)^{2} \mathrm{~d} \tilde{x}_{j} \mathrm{~d} \tilde{x}_{k}\right]\right|_{x_{i}=x_{i 1}} ^{x_{i}-x_{i 2}}\right\}=0 .
$$

The pseudoenergy is quadratic, as expected from its construction. We now show that it reduces to the small-amplitude QG pseudoenergy in the small-Rossby-number limit, $\epsilon \rightarrow 0$. In this limit, from Part 1 (2.20), the matrix of coefficients $a_{l m}$ becomes

$$
a_{l m}=\left(\begin{array}{ccc}
\tilde{z}_{z} & 0 & 0 \\
0 & \tilde{z}_{z} & 0 \\
0 & 0 & 1
\end{array}\right)
$$

while

$$
\tilde{z}_{Z} \approx(f / N)^{2}, \quad z^{\prime} \approx-\tilde{z}_{Z} Z^{\prime} \approx-g \theta^{\prime} /\left(N^{2} \theta_{0}\right),
$$

where $N(z)$ is the prescribed reference buoyancy-frequency profile of QG dynamics. The QG PV is $q \propto 1 / \sigma$, so that $\mathrm{d} q / \mathrm{d} \sigma=-q / \sigma$. In QG dynamics, we have the steady-state relation

$$
\partial_{x y}(\tilde{\Phi}, \tilde{q})=0 \Rightarrow \tilde{\Phi}=\tilde{\Phi}(\tilde{q}),
$$

where $\Phi=\phi / f$ is the QG stream function satisfying $\left(u_{g}, v_{g}\right)=\left(-\Phi_{y}, \Phi_{x}\right)$, and gradients are taken at fixed $z$. For small Rossby number we find

$$
\tilde{\Psi}_{z}=\frac{\nabla_{X} \tilde{\Psi}}{\nabla_{X} \tilde{\sigma}} \approx \frac{f \nabla_{x} \tilde{\Phi}}{\nabla_{x} \tilde{\sigma}} \approx-\frac{f^{2}}{\tilde{\sigma}} \tilde{\Phi}_{\tilde{q}}
$$

where we have used Part 1 (2.12). Since

$$
x^{\prime}=-(1 / f) v_{g}^{\prime}, \quad y^{\prime}=(1 / f) u_{g}^{\prime},
$$

we obtain, using (2.18) and the small-Rossby-number relations $(X, Y) \approx(\tilde{x}, \tilde{y})$ and $\sigma \approx z_{z} \approx(f / N)^{2}$,

$$
\lim _{\varepsilon \rightarrow 0} \mathscr{Q}_{i n t}=\frac{1}{2} \int_{D}\left[\left(u_{g}^{\prime}\right)^{2}+\left(v_{g}^{\prime}\right)^{2}+\frac{g^{2}}{\theta_{0}^{2} N^{2}}\left(\theta^{\prime 2}\right)+\frac{\tilde{\phi}_{\tilde{q}}}{f}\left(q^{\prime}\right)^{2}\right] \mathrm{d} x \mathrm{~d} y \mathrm{~d} z .
$$

Equation (2.37) is the small-amplitude Boussinesq form of the interior contribution to the QG pseudoenergy. (See equation (B 7) of MS87, noting that their stream function is also $\phi / f$. Although MS87 use a $\log$-pressure vertical coordinate $z$, the results have the same form in the pseudoheight coordinate.)

We next discuss the small-Rossby-number reduction of the boundary terms. The lateral boundary terms vanish under QG scaling (see Part 1). The $z$-boundary terms reduce as follows. Let us first assume that the $X$-components of both $\tilde{\Psi}$ and $\tilde{z}$ gradients are non-zero. Then from (2.28), for small Rossby number,

$$
\frac{\nabla \tilde{\Psi}}{\nabla \tilde{z}} \approx \frac{f^{3} \theta_{0} \hat{z} \times \tilde{u}_{g}}{g \tilde{z}_{z} \nabla_{h} \tilde{\theta}}
$$

where the gradient $\nabla_{h}=\left(\left.\partial_{x}\right|_{z},\left.\partial_{y}\right|_{z}\right)$, and we have also used Part 1 (2.9) and (2.12). The small-Rossby-number limit of $\mathscr{U}_{B}$ may then be shown to be

$$
\lim _{\varepsilon \rightarrow 0} \mathscr{U}_{s}=\left.\left[\int_{z} \frac{f}{2 \mathrm{~d} \theta_{s} / \mathrm{d} z} \frac{\hat{z} \times \tilde{u}_{g}}{\nabla_{h} \tilde{\theta}}\left(\theta^{\prime}\right)^{2} \mathrm{~d} x \mathrm{~d} y\right]\right|_{z_{1}} ^{z_{2}},
$$


where $\theta_{s}=\theta_{s}(z)$ is the prescribed reference-state potential-temperature profile of $\mathbf{Q G}$ dynamics.

We find that the expression on the right-hand side of (2.39) is the small-amplitude Boussinesq form of the boundary contribution to the QG pseudoenergy. At small amplitude and in the absence of topography, from (B 7) of MS87,

$$
\mathscr{U}_{s, Q G}=\left.\left[\int_{z}-\frac{f}{2 \mathrm{~d} \theta_{s} / \mathrm{d} z} \frac{\mathrm{d} \tilde{\Phi}}{\mathrm{d} \tilde{\theta}}\left(\theta^{\prime}\right)^{2} \mathrm{~d} x \mathrm{~d} y\right]\right|_{z_{1}} ^{z_{2}}
$$

At each $z$-boundary, $\tilde{\Phi}$ and $\tilde{\theta}$ satisfy the steady-state relation

$$
\left.\partial_{x y}\right|_{z}(\tilde{\Phi}, \tilde{\theta})=0 \Rightarrow \tilde{\Phi}=\tilde{\Phi}(\tilde{\theta}) \text { at each } z \text { surface. }
$$

To express $d \tilde{\Phi} / d \tilde{\theta}$ in terms of gradients with respect to the independent coordinates, we have, similarly to $(2.28)$,

$$
\frac{\mathrm{d} \tilde{\Phi}}{\mathrm{d} \tilde{\theta}}=\frac{\tilde{\Phi}_{x}}{\tilde{\theta}_{x}}=\frac{\tilde{\Phi}_{y}}{\tilde{\theta}_{y}}=-\frac{\hat{z} \times \tilde{u}_{g}}{\nabla_{h} \tilde{\theta}}
$$

Thus (2.39) and (2.40) are equal.

\section{Linear stability}

From (2.33) we have the following small-amplitude version of Arnol'd's first stability theorem for SG dynamics: if the matrix $a_{l m}$ is sign definite, and if $-\tilde{\Psi}_{\tilde{\sigma}}$ in the interior, $-\nabla \tilde{\Psi} / \nabla \tilde{x}_{i}$ at $x_{i}=x_{i 1}$ and $\nabla \tilde{\Psi} / \nabla \tilde{x}_{i}$ at $x_{i}=x_{i 2}$ for $i=1,2,3$ all share the same sign as the matrix everywhere in their respective regions, then the basic state is stable, since perturbations cannot grow in a mean-square sense. As discussed in $\S 2$, the matrix $a_{l m}$ is positive definite whenever the basic state is statically and inertially stable and has positive PV. These conditions are required for the physical realizability of the SG equations (Shutts \& Cullen 1987).

Since $a_{l m}$ is positive definite, we may interpret the quadratic form in (2.18) and (2.33) involving $a_{l m}$ as the energy density of the perturbation, noting also that it reduces to the QG perturbation energy (the first three terms in square brackets in (2.37)). Notice that the linear part of $E^{*}-\tilde{E}^{*}$ in the first integral in (2.17) has been eliminated by the linear part of the Casimir term, i.e. the second integral in (2.17). The SG perturbation energy is a useful quantity to know. For example, we recall that the perturbation energy is, under the WKB approximation, the quantity whose ratio with the intrinsic frequency is equal to the wave action, which is constant following a wave packet along its ray (Bretherton \& Garrett 1968).

Whether $\left(-\tilde{\Psi}_{\tilde{\sigma}}\right)$ should be positive or negative depends on the details of the basic state. For cyclonic flow around a positive PV anomaly, the term is expected to be negative. For easterly flow in a positive meridional PV gradient such as could be found on the $\beta$-plane, the term would be positive. With regard to the upper and lower boundary terms, it is evident from (2.38) and

$$
\boldsymbol{\nabla}_{h} \tilde{\theta}=-\frac{\theta_{0} f}{g} \hat{z} \times \frac{\partial \tilde{u}_{g}}{\partial z}
$$

that, in the QG limit at least, the sign of these terms depends on the sign of the ratio of a given geostrophic-wind component to its vertical derivative, i.e. its thermal-wind component. For example, a horizontal geostrophic circulation whose strength increases with height from the lower boundary and decreases with height towards the upper boundary would make the $z$-surface contribution to $\mathscr{U}$ positive. 
In Part 1, we find that lateral boundaries play a crucial role in determining the stability of a basic state. Independently of the interior basic flow, the simple presence of opposing meridional sidewalls violates the SG Charney-Stern stability criteria found in Part 1, §3. This violation of the stability criteria is associated with contributions of opposite sign to the pseudomomentum from the two meridional boundaries. Here, by contrast, it is straightforward to construct basic states which yield sign-definite lateral-boundary contributions to the pseudoenergy. As long as $\left(\tilde{x}_{i}\right)_{X_{i}}$ is positive at each lateral boundary, which is true by assumption (see (2.7)), then the lateral boundary terms in (2.33) have the same sign as the sense of the boundary geostrophic circulation, i.e. positive if cyclonic. Thus we find that the simple presence of opposing lateral boundaries does not by itself violate the stability criteria.

An interesting special case to consider is that of isovortical disturbances to a zonal linear barotropic shear flow between opposing meridional walls. In this case, the interior basic-state $\mathrm{PV}$ is constant, and $\sigma^{\prime} \equiv 0$ in the interior, so that the contributions proportional to $-\tilde{\Psi}_{\tilde{\sigma}}$ in (2.33) vanish. The remaining contributions are the positive one from the interior perturbation energy, and the meridional boundary contributions which are positive if the shear is cyclonic and negative if the shear is anticyclonic. Thus, the basic state with cyclonic shear is necessarily stable by the SG Arnol'd stability theorem, but the anticyclonic case violates the SG Arnol'd stability criteria. This is consistent with a linear stability analysis performed by Kushner (in preparation), in which unstable normal modes (albeit with exceedingly weak growth rates) are found for the anticyclonic case, but no unstable normal modes are found for the cyclonic case. We recall that both the cyclonic and the anticyclonic basic states violate the SG Charney-Stern stability criteria of Part 1.

As was noted in the Introduction, it is not clear which sign the pseudoenergy (2.17) would take for arbitrary finite-amplitude disturbances to a prescribed linearly stable basic state. In this way the finite-amplitude form of $\mathscr{U}$ is quite different from the QG invariant, which may be reduced to a relatively simple form (see, e.g. MS87, equation (B 7)) and which is clearly sign definite for a linearly stable basic state. The source of this difference is apparently the non-quadratic energy density $E^{*}$. A sign-definite invariant is necessary in order to obtain nonlinear stability, and so the small-amplitude stability theorem cannot be extended to finite amplitude at present.

\section{Local conservation law}

We now develop a local conservation law of the form

$$
\frac{\partial U_{i n t}}{\partial T}+\nabla \cdot J=0
$$

for the interior pseudoenergy density, i.e. the integrand in (2.18). Taking (4.1) as a definition of $J$ does not specify it completely: the field is only defined to within a nondivergent flux. To further fix the field's form, we require that it satisfy the groupvelocity property that in the WKB limit of a small-amplitude monochromatic wave packet propagating through a slowly varying medium,

$$
\langle J\rangle=c_{g}\left\langle U_{i n t}\right\rangle,
$$

where $c_{g}$ is the group velocity and the angular brackets denote a phase average.

The approach parallels that of Part $1, \S 7$. The linearized invertibility relation is, from (2.23),

$$
\sigma^{\prime}=h_{l m} \partial_{X_{l}} \partial_{X_{m}} \Psi^{\prime},
$$


where from (2.19)

$$
h_{l m}=-a_{l m} / f^{2} .
$$

Using the linearization of Part 1 (2.18a), and (4.3)-(4.4) and (2.25) above yields (4.1) with

$$
J=\left[\begin{array}{c}
\Psi^{\prime} x_{m T}^{\prime} a_{1 m}+\tilde{u}_{g}\left[\sigma^{\prime} \Psi^{\prime}-\frac{1}{2} \tilde{\Psi}_{\tilde{\sigma}}\left(\sigma^{\prime}\right)^{2}\right]+\frac{1}{2 f} \tilde{\sigma}_{Y}\left(\Psi^{\prime}\right)^{2} \\
\Psi^{\prime} x_{m T}^{\prime} a_{2 m}+\tilde{v}_{g}\left[\sigma^{\prime} \Psi^{\prime}-\frac{1}{2} \tilde{\Psi}_{\tilde{\sigma}}\left(\sigma^{\prime}\right)^{2}\right]-\frac{1}{2 f} \tilde{\sigma}_{X}\left(\Psi^{\prime}\right)^{2} \\
\Psi^{\prime} x_{m T}^{\prime} a_{3 m}
\end{array}\right] .
$$

At this stage the non-quadratic form of the energy has become an obstacle to generalizing these manipulations to finite amplitude. An expression in the form of (2.25) has yet to be found at finite amplitude, and such a step appears necessary to the finite-amplitude generalization. In the small-Rossby-number limit, the small-amplitude flux $J$ reduces to the small-amplitude Boussinesq QG pseudoenergy flux found in (B2b) of MS87, apart from a scaling factor of $\tilde{z}_{Z} \approx(f / N)^{2}$ in the horizontal components accounted for by the transformation from physical coordinates to IGC.

To explore the wave properties of the system, and to verify the group-velocity property, we introduce the WKB ansatz

$$
\Psi^{\prime}=\operatorname{Re}\left[\Psi^{*}(\mu X, \mu Y, \mu Z) \mathrm{e}^{\mathrm{i}(K \cdot X-\Omega T)}\right]
$$

where

$$
\mu=\frac{\text { perturbation lengthscale }}{\text { wave envelope lengthscale }} \ll 1,
$$

the dimensional wave vector $K=(K, L, M)$, and $\Omega(K, X)$ is the frequency. Substituting (4.6) into (4.5) and keeping the leading-order contributions in $\mu$ yields the phase average of $J$ :

$$
\langle J\rangle=\left[\begin{array}{c}
\Omega h_{1 m} K_{m}-\tilde{u}_{g} h_{l m} K_{l} K_{m}+\frac{1}{2 f} \tilde{\sigma}_{Y}-\frac{1}{2} \tilde{u}_{g} \tilde{\Psi}_{\tilde{\sigma}}\left(h_{l m} K_{l} K_{m}\right)^{2} \\
\Omega h_{2 m} K_{m}-\tilde{v}_{g} h_{l m} K_{l} K_{m}-\frac{1}{2 f} \tilde{\sigma}_{X}-\frac{1}{2} \tilde{v}_{\theta} \tilde{\Psi}_{\tilde{\sigma}}\left(h_{l m} K_{l} K_{m}\right)^{2} \\
\Omega h_{3 m} K_{m}
\end{array}\right] \frac{|\Psi *|^{2}}{2},
$$

where we have used the linear relations (2.24) and (4.3). The dispersion relation is found by substituting the WKB solution into the linearized governing equation and taking terms of lowest order in $\mu$. This yields

$$
\Omega=\tilde{u}_{g} K+\tilde{v}_{g} L+\frac{\left(\tilde{\sigma}_{Y} / f\right) K-\left(\tilde{\sigma}_{X} / f\right) L}{-h_{l m} K_{l} K_{m}},
$$

where the minus sign accompanies the denominator to keep the quadratic form positive. The group velocity is

$$
\begin{aligned}
c_{g(j)}=\left(\tilde{u}_{g}+\frac{\tilde{\sigma}_{Y} / f}{-h_{l m} K_{l} K_{m}}\right) \delta_{j 1}+\left(\tilde{v}_{g}-\frac{\tilde{\sigma}_{X} / f}{-h_{l m} K_{l} K_{m}}\right) \delta_{j 2} \\
+\frac{2\left[\left(\tilde{\sigma}_{Y} / f\right) K-\left(\tilde{\sigma}_{X} / f\right) L\right]}{\left(-h_{l m} K_{l} K_{m}\right)^{2}} h_{j i} K_{i},
\end{aligned}
$$

where we have used the symmetry of $h_{l m}$. 
We now sketch the demonstration of the group-velocity property, which is similar to that of Part $1, \S 7$. The phase-averaged linearized pseudoenergy is

$$
\left\langle U_{i n t}\right\rangle=-\frac{1}{4} h_{l m} K_{l} K_{m}\left(1+\tilde{\Psi}_{\tilde{\sigma}} h_{r s} K_{r} K_{s}\right)\left|\Psi^{*}\right|^{2} .
$$

If we take the third term in (4.9), multiply it by (4.10), and use $\tilde{\Psi}_{\tilde{\sigma}} \tilde{\sigma}_{Y}=\tilde{\Psi}_{Y}=-f \tilde{u}_{g}$ and $\tilde{\Psi}_{\tilde{\sigma}} \tilde{\sigma}_{X}=\tilde{\Psi}_{X}=f \tilde{v}_{g}$, the product reduces to

$$
\frac{1}{2} \Omega h_{j i} K_{i}\left|\Psi^{*}\right|^{2}
$$

for each $j$, which corresponds to the first term in each component of (4.7). As for the first term of (4.9),

$\left(\tilde{u}_{g}+\frac{\tilde{\sigma}_{Y} / f}{-h_{l m} K_{l} K_{m}}\right)\left\langle U_{i n l}\right\rangle=\frac{1}{2}\left[-\tilde{u}_{g} h_{l m} K_{l} K_{m}+\frac{\tilde{\sigma}_{Y}}{2 f}-\frac{1}{2} \tilde{u}_{g} \tilde{\Psi}_{\tilde{\sigma}}\left(h_{l m} K_{l} K_{m}\right)^{2}\right]\left|\Psi^{*}\right|^{2}$,

while a similar expression holds for the second term. These correspond to the remaining terms in (4.7). Thus, summing (4.11) with $j=1$ and (4.12) yields $\left\langle J_{(X)}\right\rangle$, and similarly for the other components of $J$, verifying the group-velocity property.

\section{5. $\beta$-plane compressible flow}

\subsection{Introduction}

In this section, we extend the results of $\$ 2-4$ to the $\beta$-plane compressible system of MSc90. The system is described in Part $1, \S 9.2$, and its conservation properties are described in Part 1, $\$ 9.4$. Like the Hoskins (1975) system, the MSc90 model is a Hamiltonian system governed by PV advection, with appropriate boundary conditions, and an invertibility principle relating the PV and boundary terms to the velocity. Its Hamiltonian structure is presented in $\$ 6$ below.

Given the similarity in structure of the two systems, many of the derivations of the results of this section closely parallel those of the previous sections. We will thus omit the details of the developments below, referring the reader to the earlier sections.

\subsection{Pseudoenergy}

Analogously to $\S 2$, we define inverse maps $\tilde{\Psi}=\tilde{\Psi}_{i n t}(\tilde{\sigma} / f, \Theta)$ and $\tilde{\Psi}=\tilde{\Psi}_{s}(\Theta)$ at the basic state. We seek $\mathscr{U}$ of the form (2.4), involving the sum of the energy and a Casimir chosen to satisfy the extremal property (2.5). Boundary variability is treated similarly to the $f$-plane system.

The energy density $E^{*}$ in IGC is defined by

with

$$
\mathscr{E}=\int E \mathrm{~d} x \mathrm{~d} y \mathrm{~d} z=\int E^{*} \mathrm{~d} X \mathrm{~d} Y \mathrm{~d} \Theta
$$

$$
E^{*}=\left[\rho\left(\frac{1}{2}\left(u_{g}^{2}+v_{g}^{2}\right)+\Theta \Psi_{\Theta}+\phi\right)-p\right] \partial_{X Y \theta}(x, y, z)=\Psi \sigma-p \partial_{X Y \Theta}(x, y, z)
$$

where in (5.2) we have used Part 1 (9.7) and (9.20) and

$$
\rho \partial_{X Y \Theta}(x, y, z)=\sigma
$$

which follows from Part 1 (9.5) and the final component of Part 1 (9.16).

The first variation of the energy is shown, in Appendix A, $\$$ A.2, to be (2.11) with $Z$ replaced by $\Theta$. The Casimir invariants have the general form given by Part 1 (9.27). The Casimir density that makes $\mathscr{U}$ extremal is

$$
C^{*}(\sigma / f, \Theta)=-\int_{[\tilde{\sigma} / f]_{s}(\theta)}^{\sigma / f} \tilde{\Psi}_{i n t}(\hat{\sigma} / f, \Theta) \mathrm{d}(\hat{\sigma} / f)-\tilde{\Psi}_{s}(\Theta)[\tilde{\sigma} / f]_{s}(\Theta)
$$


The pseudoenergy is then

$$
\begin{aligned}
\mathscr{U}=\int_{\tilde{D}}\left(E^{*}-\tilde{E}^{*}\right) \mathrm{d} X \mathrm{~d} Y \mathrm{~d} \Theta-\int_{\tilde{D}+D^{\prime}} f\left[\int_{\tilde{\sigma} / f}^{\sigma / f} \tilde{\Psi}_{i n t}(\hat{\sigma} / f, \Theta) \mathrm{d}(\hat{\sigma} / f)\right] \mathrm{d} X \mathrm{~d} Y \mathrm{~d} \Theta \\
+\int_{D^{\prime}}\left[E^{*}-\tilde{\Psi}_{i n t}(\tilde{\sigma} / f, \Theta) \tilde{\sigma}+f \int_{[\tilde{\sigma} / f]_{s}(\Theta)}^{\tilde{\sigma} / f}(\hat{\sigma} / f) \frac{\partial \tilde{\Psi}_{i n t}}{\partial(\hat{\sigma} / f)} \mathrm{d}(\hat{\sigma} / f)\right] \mathrm{d} X \mathrm{~d} Y \mathrm{~d} \Theta .
\end{aligned}
$$

The small-amplitude reduction of the interior contribution to the pseudoenergy is shown in Appendix A, §A. 2 to be

$$
\mathscr{L}_{n t}=\int_{\bar{D}} \frac{1}{2}\left[a_{l m} \Psi_{X_{l}}^{\prime} \Psi_{X_{m}}^{\prime}-f \tilde{\Psi}_{(\tilde{\sigma} / f)}\left(\sigma^{\prime} / f\right)^{2}\right] \mathrm{d} X \mathrm{~d} Y \mathrm{~d} \Theta,
$$

where we have introduced the notation $\left\{X_{l}\right\}=(X, Y, \Theta)$. The symmetric matrix of coefficients $a_{l m}$ has components

$$
\begin{aligned}
& a_{11}=-\frac{1}{g}\left[\frac{\partial_{Y \theta}(\tilde{y}, \tilde{p})}{f^{2}}-\frac{2 \beta \tilde{\Psi}_{X} \partial_{Y \theta}(\tilde{p}, \tilde{x})}{\hat{f f}^{4}}\right], \\
& a_{12}=-\frac{1}{g}\left[\frac{\partial_{\theta X}(\tilde{y}, \tilde{p})}{f^{2}}-\frac{2 \beta \tilde{\Psi}_{X} \partial_{\theta X}(\tilde{p}, \tilde{x})}{f f^{4}}\right]=-\frac{1}{g}\left[\frac{\partial_{Y \theta}(\tilde{p}, \tilde{x})}{\hat{f f}]=a_{21},}\right. \\
& a_{13}=-\frac{1}{g}\left[\frac{\partial_{X Y}(\tilde{y}, \tilde{p})}{f^{2}}-\frac{2 \beta \tilde{\Psi}_{X} \partial_{X Y}(\tilde{p}, \tilde{x})}{\hat{f} f^{4}}\right]=-\frac{1}{g}\left[-\tilde{Y} \partial_{Y \theta}(\tilde{x}, \tilde{y})\right]=a_{31}, \\
& a_{22}=-\frac{1}{g}\left[\frac{\partial_{\theta X}(\tilde{p}, \tilde{x})}{\hat{f} f}\right], \\
& a_{23}=-\frac{1}{g}\left[\frac{\partial_{X Y}(\tilde{p}, \tilde{x})}{\hat{f} f}\right]=-\frac{1}{g}\left[-\tilde{Y} \partial_{\theta X}(\tilde{x}, \tilde{y})\right]=a_{32}, \\
& a_{33}=-\frac{1}{g}\left[-\tilde{Y} \partial_{X Y}(\tilde{x}, \tilde{y})\right],
\end{aligned}
$$

and we define the basic-state quantities (cf. (A 12) and (A 17))

$$
\hat{f}=f\left[1+4 \beta f^{-3}\left(\tilde{\Psi}_{Y}-\beta f^{-3}\left(\tilde{\Psi}_{X}\right)^{2}\right)\right]^{1 / 2}, \quad \tilde{Y}=\mathrm{d} \tilde{p} / \mathrm{d} \tilde{\Psi}_{\theta}=\tilde{\rho} \Theta .
$$

The equalities for the off-diagonal terms $a_{12}$, etc. may be verified using thermal-wind type relations derived from Part 1 (9.16), e.g.

$$
\tilde{y}_{X}=\frac{1}{\hat{f} f}\left(-\tilde{\Psi}_{Y X}+2 \beta f^{-3} \tilde{\Psi}_{X} \tilde{\Psi}_{X X}\right) \text {, etc. }
$$

Defining the linearized $\sigma^{\prime}$

we find

$$
\sigma^{\prime}=-\frac{1}{g}\left[\partial_{X Y \Theta}\left(x^{\prime}, \tilde{y}, \tilde{p}\right)+\partial_{X Y \Theta}\left(\tilde{x}, y^{\prime}, \tilde{p}\right)+\partial_{X Y \Theta}\left(\tilde{x}, \tilde{y}, p^{\prime}\right)\right],
$$

$$
\begin{aligned}
\Psi^{\prime} \frac{\partial \sigma^{\prime}}{\partial T}=\frac{\partial}{\partial T}\left(\frac{1}{2} a_{l m} \Psi_{X_{l}}^{\prime} \Psi_{X_{m}}^{\prime}\right) & \\
& -\frac{1}{g}\left[\partial_{X Y \Theta}\left(\Psi^{\prime} x_{T}^{\prime}, \tilde{y}, \tilde{p}\right)+\partial_{X Y \theta}\left(\tilde{x}, \Psi^{\prime} y_{T}^{\prime}, \tilde{p}\right)+\partial_{X Y \Theta}\left(\tilde{x}, \tilde{y}, \Psi^{\prime} p_{T}^{\prime}\right)\right]
\end{aligned}
$$


The volume integral over $\tilde{D}$ of the flux terms in (5.10) is

$$
\begin{aligned}
& -\frac{1}{g} \int_{\tilde{D}}\left[\partial_{X Y \Theta}\left(\Psi^{\prime} x_{T}^{\prime}, \tilde{y}, \tilde{p}\right)+\partial_{X Y \Theta}\left(\tilde{x}, \Psi^{\prime} y_{T}^{\prime}, \tilde{p}\right)+\partial_{X Y \Theta}\left(\tilde{x}, \tilde{y}, \Psi^{\prime} p_{T}^{\prime}\right)\right] \mathrm{d} X \mathrm{~d} Y \mathrm{~d} \Theta \\
= & -\frac{1}{g}\left\{\left.\sum_{i=1}^{2}\left[\int_{x_{i}} \tilde{p}_{z} \Psi^{\prime}\left[x_{i}^{\prime}\right]_{T} \mathrm{~d} \tilde{x}_{j} \mathrm{~d} \tilde{x}_{k}\right]\right|_{x_{i}=x_{i 1}} ^{x_{i}=x_{i 2}}+\left.\left[\int_{z} \Psi^{\prime}\left(p_{T}^{\prime}-\tilde{p}_{x} x_{T}^{\prime}-\tilde{p}_{y} y_{T}^{\prime}\right) \mathrm{d} \tilde{x} \mathrm{~d} \tilde{y}\right]\right|_{z_{1}} ^{z_{2}}\right\} \\
= & \left\{\left.\sum_{i=1}^{3}\left[\int_{x_{i}} \tilde{\rho} \Psi^{\prime}\left[x_{i}^{\prime}\right]_{T} \mathrm{~d} \tilde{x}_{j} \mathrm{~d} \tilde{x}_{k}\right]\right|_{x_{i}-x_{i 1}} ^{x_{i}=x_{i 2}}\right\}-\left.\frac{\mathrm{d}}{\mathrm{d} t}\left[\int_{z} \frac{\tilde{\rho}}{2 g}\left(\Psi^{\prime}\right)^{2} \mathrm{~d} \tilde{x} \mathrm{~d} \tilde{y}\right]\right|_{z_{1}} ^{z_{2}},
\end{aligned}
$$

where we have made use of Part 1 (9.2), (9.6), (9.7), $(9.9 d)$ and (9.16). The final term in (5.11) may be rewritten as an interior contribution due to compressibility:

$$
\mathscr{U}_{c}=-\left.\left[\int_{z} \frac{\tilde{\rho}}{2 g}\left(\Psi^{\prime}\right)^{2} \mathrm{~d} \tilde{x} \mathrm{~d} \tilde{y}\right]\right|_{z_{1}} ^{z_{2}}=\left[\int_{\tilde{D}}\left(\frac{b}{2}\left(\Psi^{\prime}\right)^{2}-\frac{a_{3 l}}{\Theta} \Psi^{\prime} \Psi_{X_{l}}^{\prime}\right) \mathrm{d} X \mathrm{~d} Y \mathrm{~d} \Theta\right],
$$

where

$$
b=-\partial_{X Y \theta}(\tilde{x}, \tilde{y}, \tilde{\rho}) / g \text {. }
$$

Using a similar approach to $\$ 2.3$, if we define the boundary contribution to the pseudoenergy as

$$
\mathscr{U}_{s}=\left.\sum_{i=1}^{3}\left[\int_{x_{i}} \frac{\tilde{\rho}}{2} \frac{\nabla \tilde{\Psi}}{\nabla \tilde{x}_{i}}\left(x_{i}^{\prime}\right)^{2} \mathrm{~d} \tilde{x}_{j} \mathrm{~d} \tilde{x}_{k}\right]\right|_{x_{i}-x_{i 1}} ^{x_{i}-x_{i 2}}
$$

we find the small-amplitude conservation law for $\mathscr{U}=\mathscr{U}_{i n t}+\mathscr{U}_{c}+\mathscr{U}_{s}$ :

$$
\begin{array}{r}
\frac{\mathrm{d} \mathscr{U}}{\mathrm{d} t}=\frac{\mathrm{d}}{\mathrm{d} t}\left\{\int_{\tilde{D}} \frac{1}{2}\left[a_{l m} \Psi_{X_{l}}^{\prime} \Psi_{X_{m}}^{\prime}-2 \frac{a_{3 l}}{\Theta} \Psi^{\prime} \Psi_{X_{l}}^{\prime}+b\left(\Psi^{\prime}\right)^{2}-f \tilde{\Psi}_{(\tilde{\sigma} / f)}\left(\sigma^{\prime} / f\right)^{2}\right]\right. \\
\left.\times \mathrm{d} X \mathrm{~d} Y \mathrm{~d} \Theta+\left.\sum_{i=1}^{3}\left[\int_{x_{i}} \frac{\tilde{\rho}}{2} \frac{\nabla \tilde{\Psi}}{\nabla \tilde{x}_{i}}\left(x_{i}^{\prime}\right)^{2} \mathrm{~d} \tilde{x}_{j} \mathrm{~d} \tilde{x}_{k}\right]\right|_{x_{i}-x_{i 1}} ^{x_{i}=x_{i 2}}\right\}=0 .
\end{array}
$$

As in $\S 2.3$, we now compare (5.15) in the small-Rossby-number limit with the appropriate QG result, which in this case is the small-amplitude limit of the pseudoenergy invariant for the 'modified' QG dynamics of White (1977). White's (1977) modified scaling allows for additional compressibility effects beyond those of the standard QG scaling; these effects are also included in this SG system. The development of the finite-amplitude modified-QG invariant is presented in Appendix B. We now show that $\mathscr{U}$ in $(5.15)$ reduces to the small-amplitude form of this modifiedQG invariant, (B 9). For small Rossby number, the QG PV, $q$, is related to $\sigma$ by

(see (B 4)), yielding in this limit

$$
\frac{\sigma}{f} \approx \frac{\rho_{s}}{\mathrm{~d} \theta_{s} / \mathrm{d} z} \frac{1}{q}
$$

$$
\int_{\tilde{D}}-f \tilde{\Psi}_{(\tilde{\sigma} / f)}\left(\sigma^{\prime} / f\right)^{2} \mathrm{~d} X \mathrm{~d} Y \mathrm{~d} \Theta \approx \int_{D} \frac{\rho_{s} \tilde{\Psi}_{\tilde{q}}}{f_{0}}\left(q^{\prime}\right)^{2} \mathrm{~d} x \mathrm{~d} y \mathrm{~d} z,
$$

where $f_{0}$ is a reference value of the Coriolis parameter. In the same limit (see (2.36))

$$
\tilde{\Psi}_{\tilde{q}} \approx f \tilde{\Phi}_{\tilde{q}},
$$

where $\Phi$ is the QG stream function (B 2). 
With (5.15) and (5.17)-(5.18) we obtain

$$
\begin{array}{r}
\lim _{\theta \rightarrow 0} \mathscr{U}=\int_{D} \frac{\rho_{s}}{2}\left[\left(u_{g}^{\prime}\right)^{2}+\left(v_{g}^{\prime}\right)^{2}+\frac{1}{g} \frac{\mathrm{d} \theta_{s}}{\mathrm{~d} z}\left(\theta_{s}\left(\Psi_{\theta}^{\prime}\right)^{2}-\left(\log \rho_{s}\right)_{\theta}\left(\Psi^{\prime}\right)^{2}-2 \Psi^{\prime} \Psi_{\theta}^{\prime}\right)+\tilde{\Phi}_{\tilde{q}}\left(q^{\prime}\right)^{2}\right] \\
\times \mathrm{d} x \mathrm{~d} y \mathrm{~d} z+\left.\left[\int_{z} \frac{\rho_{s} f}{2 \mathrm{~d} \theta_{s} / \mathrm{d} z} \frac{\hat{z} \times \tilde{u}_{g}}{\nabla_{h} \tilde{\theta}}\left(\theta^{\prime}\right)^{2} \mathrm{~d} x \mathrm{~d} y\right]\right|_{z_{1}} ^{z_{z}}
\end{array}
$$

We recall that the disturbance $\Psi^{\prime}$ is a variation taken in the transformed space, and the variation $\Phi^{\prime}$ is taken in geometric coordinates. In the small-Rossby-number limit,

$$
\left.\left.\Psi^{\prime}\right|_{\theta} \approx \phi^{\prime}\right|_{\theta}+\left.\left.\Theta \Psi_{\theta}^{\prime}\right|_{\theta} \approx \phi^{\prime}\right|_{\theta}+\left.f_{0} \Phi^{\prime}\right|_{z}+\left.\frac{1}{\rho_{\delta}(z)} \frac{\mathrm{d} p_{s}}{\mathrm{~d} z} z^{\prime}\right|_{\Theta}=\left.f_{0} \Phi^{\prime}\right|_{z},
$$

where we have used (B 2) and (5.8b). With this relation it may be shown that

$$
\frac{1}{g} \frac{\mathrm{d} \theta_{s}}{\mathrm{~d} z}\left(\theta_{s}\left(\Psi_{\theta}^{\prime}\right)^{2}-\left(\log \rho_{\kappa}\right)_{\theta}\left(\Psi^{\prime}\right)^{2}-2 \Psi^{\prime} \Psi_{\theta}^{\prime}\right)=\left(\frac{f_{0}}{N}\right)^{2}\left(\Phi_{z}^{\prime}-\frac{N^{2}}{g} \Phi^{\prime}\right)^{2}+\left(\frac{f_{0}}{c}\right)^{2}\left(\Phi^{\prime}\right)^{2}
$$

where $c$ is the adiabatic sound speed. In deriving (5.21), we have also used (5.8b), (B 4) and (B 6). Substituting (5.21) into (5.19) yields (B 9).

\subsection{Linear stability}

Apart from the terms involving $\Psi^{\prime}$ in (5.15), both the conservation law and the resulting stability theorem are similar to the $f$-plane case (see (2.33)). We show that the quadratic form representing the disturbance energy in (5.15) is positive for physically meaningful basic states. The form may be written $\frac{1}{2} b_{l m} r_{l} r_{m}$, where

$$
r_{l}=\left(\Psi^{\prime}, \Psi_{X}^{\prime}, \Psi_{Y}^{\prime}, \Psi_{\Theta}^{\prime}\right)^{T}
$$

and

$$
b_{l m}=\left(\begin{array}{cccc}
b & -a_{31} / \Theta & -a_{32} / \Theta & -a_{33} / \Theta \\
-a_{31} / \Theta & a_{11} & a_{12} & a_{13} \\
-a_{32} / \Theta & a_{21} & a_{22} & a_{23} \\
-a_{33} / \Theta & a_{31} & a_{32} & a_{33}
\end{array}\right) .
$$

The conditions under which $b_{l m}$ is positive definite have the form (2.21) with the additional condition

We find that

$$
\operatorname{det} b_{l m}>0 \text {. }
$$

$$
a_{33}=\frac{\Theta \tilde{\sigma} \Theta_{\tilde{z}}}{g}, \quad \operatorname{det}\left(\begin{array}{ll}
a_{22} & a_{23} \\
a_{32} & a_{33}
\end{array}\right)=\frac{\tilde{\rho} \Theta \tilde{\sigma} \tilde{x}_{X}}{\hat{f} f g}, \quad \operatorname{det} a_{l m}=\frac{\tilde{\rho} \Theta(\tilde{\sigma})^{2}}{\hat{f} f^{3} g},
$$

and

$$
\operatorname{det} b_{l m}=-\frac{\Theta \tilde{\sigma}}{g \tilde{\rho}} \frac{\partial}{\partial \tilde{z}}\left(\frac{\tilde{\rho}}{\Theta}\right) \operatorname{det} a_{l m} .
$$

All three quantities in (5.24) are positive for inertially and statically stable basic states with $\tilde{\sigma}>0$, as in the $f$-plane case. The quantity in (5.25) is also clearly positive for statically stable states. The discussion of $\S 3$ thus holds with the interior $\sigma$ replaced by $\sigma / f$.

\subsection{Local conservation law}

We seek a local conservation law of the form

where

$$
\frac{\partial}{\partial T}\left(U_{i n t}+U_{c}\right)+\nabla \cdot J=0,
$$

$$
U_{i n t}+U_{c}=\frac{1}{2}\left[a_{l m} \Psi_{X_{l}}^{\prime} \Psi_{X_{m}}^{\prime}-2\left(a_{3 l} / \Theta\right) \Psi^{\prime} \Psi_{X_{l}}^{\prime}+b\left(\Psi^{\prime}\right)^{2}-f \tilde{\Psi}_{(\tilde{\sigma} / f)}\left(\sigma^{\prime} / f\right)^{2}\right]
$$


To determine the flux $J$, we follow the procedure of $\S 4$. Expressing $\sigma^{\prime}$ similarly to (4.3), and using (5.6)-(5.10) and

$$
x^{\prime}=-\frac{\Psi_{X}^{\prime}}{f^{2}}, \quad y^{\prime}=\frac{1}{\hat{f} f}\left(-\Psi_{Y}^{\prime}+2 \beta f^{-3} \tilde{\Psi}_{X} \Psi_{X}^{\prime}\right), \quad p^{\prime}=\tilde{Y} \Psi_{\theta}^{\prime}
$$

(cf. Part 1 (9.16), and (A 12) and (A 18) below) we obtain

$$
J=\left[\begin{array}{c}
\Psi^{\prime}\left[-\Psi_{X_{m} T}^{\prime} a_{1 m}+\frac{1}{\Theta} a_{13} \Psi_{T}^{\prime}\right]-\frac{1}{f} \tilde{\Psi}_{Y}\left[\sigma^{\prime} \Psi^{\prime \prime}-\frac{1}{2} f \tilde{\Psi}_{(\tilde{\sigma} / f)}\left(\sigma^{\prime} / f\right)^{2}\right]+\frac{1}{2}(\tilde{\sigma} / f)_{Y}\left(\Psi^{\prime}\right)^{2} \\
\Psi^{\prime}\left[-\Psi_{X_{m} T}^{\prime} a_{2 m}+\frac{1}{\Theta} a_{23} \Psi_{T}^{\prime}\right]+\frac{1}{f} \tilde{\Psi}_{X}\left[\sigma^{\prime} \Psi^{\prime}-\frac{1}{2} f \tilde{\Psi}_{(\tilde{\sigma} / f)}\left(\sigma^{\prime} / f\right)^{2}\right]-\frac{1}{2}(\tilde{\sigma} / f)_{X}\left(\Psi^{\prime}\right)^{2} \\
\Psi^{\prime}\left[-\Psi_{X_{m} T}^{\prime} a_{3 m}+\frac{1}{\Theta} a_{33} \Psi_{T}^{\prime}\right]
\end{array}\right]
$$

which should be compared to (4.5). Apart from scaling factors from the coordinate transformation, (5.28) reduces at small Rossby number to the small-amplitude form of the modified-QG pseudoenergy flux. The finite-amplitude form of the QG flux is (B 11).

Using the WKB ansatz (4.6) with $Z$ replaced by $\Theta$, to lowest order in $\mu$

$$
\langle J\rangle=\left[\begin{array}{c}
-\Omega a_{1 m} K_{m}-\frac{1}{f} \tilde{\Psi}_{Y} a_{l m} K_{l} K_{m}+\frac{1}{2}(\tilde{\sigma} / f)_{Y}+\frac{1}{2 f^{2}} \tilde{\Psi}_{Y} \tilde{\Psi}_{(\tilde{\sigma} / f)}\left(a_{l m} K_{l} K_{m}\right)^{2} \\
-\Omega a_{2 m} K_{m}+\frac{1}{f} \tilde{\Psi}_{X} a_{l m} K_{l} K_{m}-\frac{1}{2}(\tilde{\sigma} / f)_{X}-\frac{1}{2 f^{2}} \tilde{\Psi}_{X} \tilde{\Psi}_{(\tilde{\sigma} / f)}\left(a_{l m} K_{l} K_{m}\right)^{2} \\
-\Omega a_{3 m} K_{m}
\end{array}\right] \frac{\left|\Psi^{*}\right|^{2}}{2},
$$

where we have used Part 1 (9.16) and (5.7) above, and note that the phase average of the compressibility terms proportional to $\Psi_{T}^{\prime}$ in (5.28) vanishes.

The dispersion relation is

$$
\Omega=-\frac{\Psi_{Y}}{f} K+\frac{\Psi_{X}}{f} L+\frac{(\tilde{\sigma} / f)_{Y} K-(\tilde{\sigma} / f)_{X} L}{a_{l m} K_{l} K_{m}},
$$

and the group velocity

$$
c_{g(j)}=\left(-\frac{\tilde{\Psi}_{Y}}{f}+\frac{(\tilde{\sigma} / f)_{Y}}{a_{l m} K_{l} K_{m}}\right) \delta_{j 1}+\left(\frac{\tilde{\Psi}_{X}}{f}-\frac{(\tilde{\sigma} / f)_{X}}{a_{l m} K_{l} K_{m}}\right) \delta_{j 2}-\frac{2\left[(\tilde{\sigma} / f)_{Y} K-(\tilde{\sigma} / f)_{X} L\right]}{\left(a_{l m} K_{l} K_{m}\right)^{2}} a_{j i} K_{i}
$$

The verification of the group-velocity property is analogous to that of $\S 4$, with the proviso that the vertical wavelength be much shorter than the geometric mean of the density and the potential-temperature scale heights.

\section{Conservation laws and Hamiltonian structure}

In this section we show how the zonal impulse, energy and Casimir invariants of SG dynamics arise naturally from Noether's theorem for a Hamiltonian system. To do so we cast the Hamiltonian structure of SG dynamics in Eulerian form, as Roulstone \& 
Norbury (1994) have done independently for the $f$-plane Boussinesq system, using a geometric approach (see also Kushner 1993). This is to be distinguished from the Lagrangian formulation of SG dynamics discussed by Salmon $(1985,1988)$. We present both the $f$-plane Boussinesq and $\beta$-plane compressible systems' Hamiltonian structure. Our discussion will centre on the interior dynamics, although the problem of incorporating boundary dynamics will be discussed.

The usual procedure to obtain the Eulerian form is as follows (e.g. Shepherd 1990). The globally conserved energy $\mathscr{E}$ is taken to be the Hamiltonian $\mathscr{H}$. A vector function $u$ comprising the system's dynamical fields is defined and the equations of motion are written

$$
u_{t}=J \frac{\delta \mathscr{H}}{\delta u},
$$

where $J=J_{i j}$ is the skew-symmetric Poisson operator satisfying a generalized 'product rule' and Jacobi's identity. Variational derivatives on the Hamiltonian are defined with respect to an appropriate inner product.

If boundary dynamics are neglected, then $u=\sigma$ is the sole dynamical variable in the SG system, and from Part $1(2.18 a)$ and (2.19), and the first variation of $\mathscr{E}=\mathscr{H}$ (equation (2.11) above, with the boundary terms neglected), (6.1) has the specific form

$$
\frac{\partial \sigma}{\partial T}=J \frac{\delta \mathscr{H}}{\delta \sigma},
$$

where on the $f$-plane

$$
J=(1 / f) \partial_{X Y}(\sigma, \cdot) .
$$

Under these restricted boundary conditions, the structure is analogous to that of QG interior flow (e.g. Shepherd 1990), with $\sigma$ replaced by the scaled QG $q$, and a minus sign appearing in the Poisson operator since $\sigma \sim 1 / q$. The definition of the inner product and the proof of Jacobi's identity are also similar.

Systems in the form (6.1) have two types of globally conserved invariants: those corresponding to explicit continuous symmetries (through Noether's theorem), and the so-called Casimir invariants corresponding to degeneracies in the Poisson bracket. The latter are functionals $\mathscr{C}$ satisfying

$$
J \frac{\delta \mathscr{C}}{\delta \sigma}=\mathbf{0} .
$$

The quantities identified as $\mathscr{C}$ in Part 1 (4.11) indeed satisfy (6.4). By Noether's theorem, we obtain a conserved zonal impulse or linear momentum corresponding to the system's zonal symmetry by seeking a functional $\mathscr{M}$ such that

$$
J \frac{\delta \mathscr{M}}{\delta \sigma}=-\sigma_{X} .
$$

Equation (6.5) is evidently satisfied by

$$
\mathscr{M}=\int-f Y \sigma \mathrm{d} X \mathrm{~d} Y \mathrm{~d} Z .
$$

This recovers Part 1 (4.9) with Part 1 (4.10).

On the $\beta$-plane we again choose $u=\sigma$, and since (2.11) holds with $Z$ replaced by $\Theta$, we obtain (6.2) with

$$
J=\partial_{X Y}(\sigma / f, \cdot) .
$$


It is straightforward to show that the Casimirs for the system are of the form Part 1 (9.27), and that the zonal impulse has the form found in Part 1 (9.26):

$$
\mathscr{M}=\int-F(Y) \sigma \mathrm{d} X \mathrm{~d} Y \mathrm{~d} \Theta
$$

where $F(Y)$ is defined by

$$
\mathrm{d} F / \mathrm{d} Y=f(Y) \text {. }
$$

We wish to emphasize the utility of the Hamiltonian approach in obtaining the invariants, in particular the zonal impulse invariants $\mathscr{M}$. Although analogous to the QG zonal impulse, whose interior contribution is $\int \rho y q \mathrm{~d} x \mathrm{~d} y \mathrm{~d} z$, the invariants, in particular (6.8), are neither 'obvious' nor have been found, to our knowledge, in previous studies. It is easily shown that the energy $\mathscr{E}=\mathscr{H}$ is the invariant corresponding to the time symmetry in the equations.

While the first variation on the energy $\mathscr{E}=\mathscr{H}$ in $(2.11)$ includes boundary terms, we have not yet determined a structure that includes the boundary dynamics and can be shown to satisfy Jacobi's identity. There are, however, strong parallels with QG dynamics, such as the role of $\theta$ at the $z$-boundaries in both systems. Since virtually all energy-conserving fluid systems have proven to be Hamiltonian, we believe these difficulties to be essentially technical, and take the structure to exist. Those results that include boundary contributions in this study do not rely on the explicit form of the Hamiltonian structure, but on the conservation laws which follow from it.

\section{Discussion}

We have derived the pseudoenergy invariant for SG dynamics, and have used it to extend some pseudoenergy-based results of QG dynamics to SG dynamics: Arnol'd's first stability theorem for small disturbances to steady flows, and a local wave-activity conservation law describing the evolution of small disturbances to these flows. The existence of the invariant, and its similarity at small amplitude to the QG form, is guaranteed because SG dynamics, like QG dynamics, is a PV-advecting invertible system with an underlying Hamiltonian structure.

At finite amplitude, the SG pseudoenergy, unlike the QG invariant, does not have obvious sign-definite properties. This is in contrast to the interior part of the SG pseudomomentum, which is similar to that of QG (see Part $1, \S 5$ ), and it arises from the complicated nonlinear invertibility relation of the SG system. At small amplitude, however, the QG and SG interior pseudoenergies are analogous. The ageostrophic effects allowed at lateral boundaries yield contributions to the pseudoenergy that are absent in the QG conservation law.

Conservation of the small-amplitude form of the pseudoenergy yields a smallamplitude version of Arnol'd's first stability theorem. The QG theorem describes conditions on the derivative of the geostrophic stream function with respect to the interior PV and to the boundary potential temperature that must be satisfied in order for the system to be stable. The SG theorem is shown to be analogous, although the new lateral-boundary contributions to the pseudoenergy yield additional stability criteria, and the boundary criteria are expressed in terms of basic-state gradients on potential-temperature surfaces rather than on the material surfaces themselves. Unlike the case with the SG Charney-Stern stability theorem (see Part 1), the presence of lateral boundaries does not automatically violate the stability criteria. For the special case of a barotropic linear shear flow, violation of the Arnol'd stability criteria 
corresponds directly to a weak barotropic normal-mode instability (Kushner, in preparation). Because the pseudoenergy invariant is not self-evidently sign definite at finite amplitude, we have not been able to construct a nonlinear Liapunov stability theorem. This is an unsatisfactory aspect of the analysis.

A local flux law has been found for the small-amplitude pseudoenergy, but we have not been able to construct such a flux law for finite-amplitude disturbances. The local form of the conservation law is similar to the small-amplitude form of the QG law. The pseudoenergy flux has been shown to satisfy the group-velocity property in the WKB limit.

The Hamiltonian structure of QG dynamics and SG dynamics is analogous for the interior dynamics. The zonal impulse, energy and Casimir functionals are all invariants corresponding to symmetries in the Hamiltonian structure. The problem of the boundary contributions to the Hamiltonian structure has not yet been resolved, in the sense that the dynamical equations at the boundaries are not explicitly represented. However, it must be emphasized that the interior Hamiltonian structure does generate the full set of conserved quantities needed to construct the boundary contributions to the pseudomomentum (Part 1 (5.13)) and the pseudoenergy (2.17). We hope that the insight gained by considering the variational problem at the boundaries will provide a first step towards obtaining the full Hamiltonian structure of the system.

P.J.K. is supported by a postgraduate fellowship from the Natural Sciences and Engineering Research Council of Canada (NSERC). T.G.S. is supported by NSERC as well as by the Atmospheric Environment Service of Canada.

\section{Appendix A. First and second variations on the energy functional}

\section{A.1. f-plane Boussinesq system}

In this subsection we derive equations (2.11) and (2.18). From (2.6),

$$
\delta \mathscr{E}=\int_{\tilde{D}} \delta E^{*} \mathrm{~d} X \mathrm{~d} Y \mathrm{~d} Z+\left.\sum_{i=1}^{3}\left[\left.\int_{x_{i}, \tilde{D}} E^{*} \delta X_{i}\right|_{x_{i}} \mathrm{~d} X_{j} \mathrm{~d} X_{k}\right]\right|_{x_{i}-x_{i 1}} ^{x_{i}-x_{i 2}} .
$$

From Part 1 (2.12), and (2.8) and (2.10) above,

$$
\begin{aligned}
\delta E^{*} & =f^{2}\{(x-X) \delta x+(y-Y) \delta y-Z \delta z\} \sigma+(\Psi-\phi) \delta \sigma \\
& =-\sigma \phi_{x_{i}} \delta x_{i}+\Psi \delta \sigma-\phi \delta \sigma \\
& =\Psi \delta \sigma+\sum_{i=1}^{3} \partial_{X Y Z}\left(-\phi \delta x_{i}, x_{j}, x_{k}\right)
\end{aligned}
$$

where

$$
\delta \sigma=\sum_{i=1}^{3} \partial_{X Y Z}\left(\delta x_{i}, x_{j}, x_{k}\right) .
$$

Substituting (A 2) into (A 1) yields to first order

$$
\begin{aligned}
\delta \mathscr{E}=\int_{\tilde{D}} \Psi \delta \sigma \mathrm{d} X \mathrm{~d} Y \mathrm{~d} Z+\sum_{i=1}^{3}\left[\int_{x_{i}, \tilde{D}}-\phi \delta x_{i} \partial_{X_{j} X_{k}}\left(x_{j}, x_{k}\right) \mathrm{d} X_{j} \mathrm{~d} X_{k}\right. \\
\left.\quad+\int_{x_{i}, \tilde{D}} E^{*} \delta X_{i} \mid \bar{x}_{i} \mathrm{~d} X_{j} \mathrm{~d} X_{k}\right]\left.\right|_{x_{i}-x_{i 1}} ^{x_{i}-x_{i 2}} .
\end{aligned}
$$

Using the relations $\delta x_{i}=-\left.\left(x_{i}\right)_{X_{i}} \delta X_{i}\right|_{x_{i}}$, which require the conditions (2.7) to hold, gives

$$
\int_{x_{i}, \tilde{D}}-\phi \delta x_{i} \partial_{X_{j} X_{k}}\left(x_{j}, x_{k}\right) \mathrm{d} X_{j} \mathrm{~d} X_{k}=\left.\int_{x_{i}, \tilde{D}} \phi \sigma \delta X_{i}\right|_{x_{i}} \mathrm{~d} X_{j} \mathrm{~d} X_{k} .
$$


Substituting (A 5) into (A 4), and using the second equality of (2.10), (2.11) follows.

We now develop equation (2.18). The energy density may be expanded to second order as

$$
E^{*}=\tilde{E}^{*}+\delta E^{*}+\delta^{2} E^{*},
$$

where $\tilde{E}^{*}$ denotes $E^{*}$ evaluated at the basic state, $\delta E^{*}$ is given by (A 2), and the secondorder contribution is

$$
\begin{aligned}
\delta^{2} E^{*}=f^{2}\left\{\frac{1}{2}\left[(\delta x)^{2}+(\delta y)^{2}\right] \sigma+[\delta x(x-X)\right. & +\delta y(y-Y)-Z \delta z] \delta \sigma \\
& \left.+\left(\frac{1}{2}\left[(x-X)^{2}+(y-Y)^{2}\right]-Z z\right) \delta^{2} \sigma\right\},
\end{aligned}
$$

with

$$
\delta^{2} \sigma=\sum_{i=1}^{3} \partial_{X Y Z}\left(\delta x_{i}, \delta x_{j}, x_{k}\right) .
$$

The terms in $\delta^{2} E^{*}$ may be handled in a similar manner to the manipulations in (A 2), i.e. by using Part 1 (2.12). We find

$$
E^{*}-\tilde{E}^{*}=\tilde{\Psi}\left(\delta \sigma+\delta^{2} \sigma\right)+\frac{1}{2} f^{2} a_{l m} \delta x_{l} \delta x_{m}+\nabla \cdot D,
$$

where the tildes have now been added to denote basic-state quantities explicitly. In (A 9) the matrix $a_{l m}$ is given by (2.19), and the divergence term is defined by

$$
\begin{aligned}
-\nabla \cdot \boldsymbol{D}=\sum_{i=1}^{3}\left[\partial_{X Y Z}\left(\tilde{\phi} \delta x_{i}, \tilde{x}_{j}, \tilde{x}_{k}\right)+\frac{1}{2}\left\{\partial_{X Y Z}\left(\tilde{\phi} \delta x_{i}, \delta x_{j}, \tilde{x}_{k}\right)\right.\right. \\
\\
\left.\left.+\partial_{X Y Z}\left(\delta x_{i}, \tilde{\phi} \delta x_{j}, \tilde{x}_{k}\right)+\partial_{X Y Z}\left(\tilde{\phi}_{\tilde{x}_{l}} \delta x_{l} \delta x_{i}, \tilde{x}_{j}, \tilde{x}_{k}\right)\right\}\right] .
\end{aligned}
$$

From (2.15), the Casimir term is to second order

$$
C^{*}-\tilde{C}^{*}=-\tilde{\Psi}_{i n t}\left(\delta \sigma+\delta^{2} \sigma\right)-\frac{1}{2} \frac{\partial \tilde{\Psi}_{i n t}}{\partial \tilde{\sigma}}(\delta \sigma)^{2} .
$$

Since $\tilde{\Psi}_{i n t}=\tilde{\Psi}$ at the basic state, the interior contribution to the integral over the sum of (A 9) and (A 11) is indeed (2.18), with the $\delta$ replaced by the prime notation.

\section{A.2. $\beta$-plane compressible system}

Here we show that (2.11) holds for the $\beta$-plane compressible equations with $Z$ replaced by $\Theta$, and also that the small-amplitude interior contribution to the pseudoenergy is (5.6). From (5.2)

$$
\begin{aligned}
\delta E^{*} & =\Psi \delta \sigma+\sigma\left(u_{g} \delta u_{g}+v_{g} \delta v_{g}+\Theta \delta \Psi_{\theta}+\delta \phi\right)-\delta p \partial_{X Y \theta}(x, y, z)-p \sum_{i=1}^{3} \partial_{X Y \Theta}\left(\delta x_{i}, x_{j}, x_{k}\right) \\
& =\Psi \delta \sigma+\frac{\sigma}{\rho}\left(-p_{x} \delta x-p_{y} \delta y\right)+\sigma \delta \phi-p \sum_{i=1}^{3} \partial_{X Y \theta}\left(\delta x_{i}, x_{j}, x_{k}\right) \\
& =\Psi \delta \sigma-\sum_{i=1}^{3} \partial_{X Y \theta}\left(p \delta x_{i}, x_{j}, x_{k}\right),
\end{aligned}
$$

where we have used Part 1 (9.2) and (9.6), and (5.3) above, and

$$
\delta p=\gamma \delta \Psi_{\theta}=\rho \Theta \delta \Psi_{\theta},
$$

where $Y$ is defined in Part 1 (9.35). Equation (A 12) follows from Part 1 (9.16). To obtain the first variation, the boundary terms are treated similarly to (A 5).

In taking the second variation on $\mathscr{U}$, extra algebraic complication is added by the 
need to expand $p$ and $y$ to first and second order in the disturbance stream function $\Psi^{\prime}$. We use the expansions

$$
y=\tilde{y}+\delta y+\delta^{2} y, \quad p=\tilde{p}+\delta p+\delta^{2} p,
$$

which may be expressed in terms of the stream function as follows. For $y$, using Part 1 (9.16),

with

$$
y=Y+(f / 2 \beta)\left[1-(1+a)^{1 / 2}\right]
$$

Thus

$$
\left.\begin{array}{c}
a=4 \beta f^{-3}\left(\Psi_{Y}-\beta f^{-3}\left(\Psi_{x}\right)^{2}\right), \quad \delta a=4 \beta f^{-3}\left(\delta \Psi_{Y}-2 \beta f^{-3} \tilde{\Psi}_{X} \delta \Psi_{X}\right) \\
\delta^{2} a=-4\left(\beta f^{-3}\right)^{2}\left(\delta \Psi_{X}\right)^{2}
\end{array}\right\}
$$

$$
\delta y=\frac{\mathrm{d} \tilde{y}}{\mathrm{~d} \tilde{a}} \delta a, \quad \delta^{2} y=\frac{\mathrm{d} \tilde{y}}{\mathrm{~d} \tilde{a}} \delta^{2} a+\frac{1}{2} \frac{\mathrm{d}^{2} \tilde{y}}{\mathrm{~d} \tilde{a}^{2}}(\delta a)^{2} .
$$

If we define

from (A 14), then

$$
\hat{f}=f(1+\tilde{a})^{1 / 2}=f\left[1-2 \beta f^{-1}(\tilde{y}-Y)\right],
$$

$$
\delta y=-(1 / f \hat{f})\left(\delta \Psi_{Y}-2 \beta f^{-3} \tilde{\Psi}_{X} \delta \Psi_{X}\right)
$$

For $p, \delta p$ is given by (A 12) and

$$
\delta^{2} p=\frac{1}{2} \frac{\mathrm{d} \tilde{Y}}{\mathrm{~d} \tilde{\Psi}_{\theta}}\left(\delta \Psi_{\theta}\right)^{2}
$$
have

The second variation is found by taking $\delta^{2} E^{*}$ similarly to (A 7). Instead of (A 8), we

$$
\begin{aligned}
\delta^{2} \sigma=-(1 / g)\left[\partial_{X Y \Theta}(\delta x, \delta y, \tilde{p})+\partial_{X Y \Theta}(\tilde{x}, \delta y, \delta p)+\partial_{X Y \Theta}(\delta x, \tilde{y}, \delta p)\right. \\
\left.+\partial_{X Y \Theta}\left(\tilde{x}, \delta^{2} y, \tilde{p}\right)+\partial_{X Y \Theta}\left(\tilde{x}, \tilde{y}, \delta^{2} p\right)\right] .
\end{aligned}
$$

We also make use of the following relations, which are obtained using Part 1 (9.16) and (A 12), (A 16) and (A 18) above:

$$
\left.\begin{array}{l}
(\delta x)^{2}=\frac{\left(\delta \Psi_{X}\right)^{2}}{f^{4}}, \quad \delta x \delta y=\frac{\delta \Psi_{X} \delta \Psi_{Y}}{\hat{f} f^{3}}-\frac{2 \beta f^{-3} \tilde{\Psi}_{X}\left(\delta \Psi_{X}\right)^{2}}{\hat{f} f^{3}}, \\
(\delta y)^{2}=\frac{\left(\delta \Psi_{Y}\right)^{2}}{\hat{f}^{2} f^{2}}-\frac{4 \beta f^{-3} \tilde{\Psi}_{X} \delta \Psi_{X} \delta \Psi_{Y}}{\hat{f}^{2} f^{2}}+\frac{\left(2 \beta f^{-3} \tilde{\Psi}_{X} \delta \Psi_{X}\right)^{2}}{\hat{f}^{2} f^{2}}, \\
\delta y \delta p=-\frac{\tilde{Y} \delta \Psi_{Y} \delta \Psi_{\theta}}{\hat{f} f}+\frac{2 \tilde{Y} \beta f^{-3} \tilde{\Psi}_{X} \delta \Psi_{\theta} \delta \Psi_{X}}{\hat{f f f}}, \quad \delta p \delta x=-\frac{\tilde{Y} \delta \Psi_{\theta} \delta \Psi_{X}}{f^{2}} .
\end{array}\right\}
$$

After some manipulation, we find

$$
E^{*}-\tilde{E}^{*}=\tilde{\Psi}\left(\delta \sigma+\delta^{2} \sigma\right)+\frac{1}{2} a_{l m} \delta \Psi_{X_{l}} \delta \Psi_{X_{m}}+\nabla \cdot D
$$

where $a_{l m}$ is given by (5.7) and the divergence term is

$$
\begin{aligned}
g \boldsymbol{\nabla} \cdot D= & g \boldsymbol{\nabla} \cdot \boldsymbol{R}+\partial_{X Y \Theta}(\tilde{\phi} \delta x, \tilde{y}, \tilde{p})+\partial_{X Y \Theta}\left(\tilde{x}, \tilde{\phi}\left[\delta y+\delta^{2} y\right], \tilde{p}\right)+\partial_{X Y \Theta}\left(\tilde{x}, \tilde{y}, \tilde{\phi}\left[\delta p+\delta^{2} p\right]\right) \\
& +\frac{1}{2}\left[\partial_{X Y \Theta}(\tilde{\phi} \delta x, \delta y, \tilde{p})+\partial_{X Y \Theta}(\delta x, \tilde{\phi} \delta y, \tilde{p})+\partial_{X Y \Theta}(\tilde{x}, \tilde{\phi} \delta y, \delta p)\right. \\
& +\partial_{X Y \Theta}(\tilde{x}, \delta y, \tilde{\phi} \delta p)+\partial_{X Y \Theta}(\delta x, \tilde{y}, \tilde{\phi} \delta p)+\partial_{X Y \Theta}(\tilde{\phi} \delta x, \tilde{y}, \delta p) \\
& +\partial_{X Y \Theta}\left(f^{2} \delta x[(X-\tilde{x}) \delta x+(Y-\tilde{y}) \delta y], \tilde{y}, \tilde{p}\right)+\partial_{X Y \Theta}\left(-\Theta \delta p \delta x, \tilde{y}, \tilde{\Psi}_{\Theta}\right) \\
& +\partial_{X Y \Theta}\left(\tilde{x}, f^{2} \delta y[(X-\tilde{x}) \delta x+(Y-\tilde{y}) \delta y], \tilde{p}\right)+\partial_{X Y \Theta}\left(\tilde{x},-\Theta \delta y \delta p, \tilde{\Psi}_{\Theta}\right) \\
& \left.+\partial_{X Y \Theta}\left(\tilde{x}, \tilde{y}, f^{2} \delta p[(X-\tilde{x}) \delta x+(Y-\tilde{y}) \delta y]-\Theta \tilde{Y}^{-1}(\delta p)^{2}\right)\right],
\end{aligned}
$$

with $\boldsymbol{\nabla} \cdot \boldsymbol{R}$ the first- and second-order contributions to $-\partial_{X Y \Theta}(x, y, \phi p) / g$. 
The Casimir terms are treated as in $\S \mathbf{A} .1$, and the sum of the Casimir and energy terms yields (5.6).

\section{Appendix B. Pseudoenergy invariant for White's modified QG equations}

In this section we derive the finite-amplitude pseudoenergy invariant for the modified QG equations of White (1977). White obtains the modified QG equations by relaxing the standard QG scaling assumption that $N^{2} H / g \ll 1$, where $H$ is a typical vertical scale of motion. Since this assumption breaks down for planetary-scale compressible flow, White takes $N^{2} H / g=O(1)$. With the new scaling, the evolution equations governing the system are

$$
\begin{aligned}
\mathrm{D} q / \mathrm{D} t=0 & \text { in the interior, } \\
u_{g}=0 & \text { at } x=x_{1}, x_{2}, \\
v_{g}=0 & \text { at } y=y_{1}, y_{2}, \\
\mathrm{D} \theta / \mathrm{D} t=0 & \text { at } z=z_{1}, z_{2},
\end{aligned}
$$

for the same domain geometry as in the body of this paper. (One may also replace (B $1 b$ ) by periodicity of all fields in $x$.) In (B 1) $z$ is the geometric height coordinate and $\Phi$ is the QG stream function given by

$$
\Phi=\left(p-p_{s}\right) /\left(f_{0} \rho_{s}\right)
$$

where $p-p_{s}$ is the pressure deviation from the reference pressure $p_{8}(z), f_{0}$ is a reference value of the Coriolis parameter, and $\rho_{s}=\rho_{\delta}(z)$ is the reference density profile. The material derivative is

$$
\mathrm{D} / \mathrm{D} t=\partial_{t}+\partial_{x y}(\Phi, \cdot)
$$

and the diagnostic invertibility relations are

$$
\left(u_{g}, v_{g}, \theta, q\right)=\left(-\Phi_{y}, \Phi_{x}, \frac{\theta_{s} f_{0}}{g}\left[\Phi_{z}-\frac{N^{2}}{g} \Phi\right], \quad \nabla_{h}^{2} \Phi+f+\frac{1}{\rho_{s}} \frac{\partial}{\partial z}\left[\frac{\rho_{s} f_{0}^{2}}{N^{2}} \frac{\partial \Phi}{\partial z}\right]\right),
$$

where $f=f_{0}+\beta y$ is the Coriolis parameter on the $\beta$-plane.

The principal change to the standard QG equations due to the modified scaling is the redefinition of $\theta$, the deviation from the reference potential temperature profile $\theta_{\delta}(z)$. In the standard QG set, the term involving $N^{2} \Phi / g$ in the definition of $\theta$ in (B 4) is neglected.

The globally conserved energy for the system is

$$
\mathscr{E}=\frac{1}{2} \int_{D} \rho_{s}\left[\left|\nabla_{h} \Phi\right|^{2}+\left(\frac{f_{0}}{N}\right)^{2}\left(\Phi_{z}-\frac{N^{2}}{g} \Phi\right)^{2}+\left(\frac{f_{0}}{c}\right)^{2} \Phi^{2}\right] \mathrm{d} x \mathrm{~d} y \mathrm{~d} z
$$

where $c$ is the adiabatic sound speed satisfying

$$
\left(\log \rho_{s}\right)_{z}=-\left(\left(N^{2} / g\right)+\left(g / c^{2}\right)\right) \text {. }
$$

The last term in the integrand of (B 5) represents a contribution to the energy due to compressibility (White 1977).

The Casimirs of the system have the form

$$
\mathscr{C}=\int_{D} \rho_{s} C(q ; z) \mathrm{d} x \mathrm{~d} y \mathrm{~d} z+\int_{z_{1}} \rho_{8} F_{1}(\theta) \mathrm{d} x \mathrm{~d} y+\int_{z_{2}} \rho_{8} F_{2}(\theta) \mathrm{d} x \mathrm{~d} y,
$$

where $C, F_{1}$ and $F_{2}$ are arbitrary functions of their arguments. 
Manipulations similar to those found in MS87 and $\$ 2$ yield the pseudoenergy $\mathscr{U}$ for finite-amplitude disturbances to steady states:

$$
\begin{aligned}
\mathscr{U}= & \int_{D} \rho_{s}\left\{\frac{1}{2}\left[\left|\nabla_{h} \Phi^{\prime}\right|^{2}+\left(\frac{f_{0}}{N}\right)^{2}\left(\Phi_{z}^{\prime}-\frac{N^{2}}{g} \Phi^{\prime}\right)^{2}+\left(\frac{f_{0}}{c}\right)^{2}\left(\Phi^{\prime}\right)^{2}\right]\right. \\
& \left.+\int_{0}^{q^{\prime}}\left[\tilde{\Phi}_{i n t}(\tilde{q}+\hat{q} ; z)-\tilde{\Phi}_{i n t}(\tilde{q} ; z)\right] \mathrm{d} \hat{q}\right\} \mathrm{d} x \mathrm{~d} y \mathrm{~d} z \\
& -\left[\int_{z_{i}}\left\{\frac{\rho_{s} f_{0}}{\mathrm{~d} \theta_{s} / \mathrm{d} z} \int_{0}^{\theta^{\prime}}\left[\tilde{\Phi}_{z_{i}}(\tilde{\theta}+\hat{\theta})-\tilde{\Phi}_{z_{i}}(\tilde{\theta})\right] \mathrm{d} \hat{\theta}\right\} \mathrm{d} x \mathrm{~d} y\right]_{z_{1}}^{z_{2}},
\end{aligned}
$$

where $\tilde{\Phi}_{i n t}$ and $\tilde{\Phi}_{z_{i}}$ are the mappings, defined at the basic state, between $\tilde{\Phi}$ and $\tilde{q}$ in the interior and between $\tilde{\Phi}$ and $\tilde{\theta}$ at the boundary (see $\$ 2.1$ ). Apart from the terms involving $N^{2} \Phi^{\prime} / g$ and $\left(\Phi^{\prime}\right)^{2}$ in the disturbance energy, (B 8) is identical to the QG pseudoenergy given by (B 7) of MS87.

The small-amplitude reduction of $\mathscr{U}$ is

$$
\begin{aligned}
\mathscr{U} \approx \frac{1}{2} \int_{D} \rho_{s}\left\{\left[\left|\nabla_{h} \Phi^{\prime}\right|^{2}+\left(\frac{f_{0}}{N}\right)^{2}\left(\Phi_{z}^{\prime}-\frac{N^{2}}{g} \Phi^{\prime}\right)^{2}+\left(\frac{f_{0}}{c}\right)^{2}\left(\Phi^{\prime}\right)^{2}\right]\right. \\
\left.+\frac{\mathrm{d} \tilde{\Phi}}{\mathrm{d} \tilde{q}}\left(q^{\prime}\right)^{2}\right\} \mathrm{d} x \mathrm{~d} y \mathrm{~d} z-\left[\int_{z_{i}} \frac{\rho_{s} f_{0}}{2 \mathrm{~d} \theta_{s} / \mathrm{d} z} \frac{\mathrm{d} \tilde{\Phi}}{\mathrm{d} \tilde{\theta}}\left(\theta^{\prime}\right)^{2} \mathrm{~d} x \mathrm{~d} y\right]_{z_{1}}^{z_{2}} .
\end{aligned}
$$

Equation (B 9), valid for steady (and in general non-parallel) basic states, is the generalization of the invariant found in Blumen (1978) for zonal basic states.

Defining the total interior pseudoenergy density $U_{i n t}+U_{c}$ as the integrand of the first integral in (B 8), we find

$$
(\partial / \partial t)\left(U_{i n t}+U_{c}\right)+\nabla \cdot J=0
$$

where the finite-amplitude pseudoenergy flux is

$$
\begin{aligned}
J=\rho_{s}\left\{\left(\int _ { 0 } ^ { q ^ { \prime } } \left[\tilde{\Phi}_{i n t}(\tilde{q}\right.\right.\right. & \left.\left.+\hat{q} ; z)-\tilde{\Phi}_{i n t}(\tilde{q} ; z)\right] \mathrm{d} \hat{q}-\Phi^{\prime} q^{\prime}\right) u_{g} \\
& \left.-\Phi^{\prime} \frac{\partial}{\partial t}\left[\nabla_{h} \Phi^{\prime}+\left(\frac{f_{0}}{N}\right)^{2}\left(\Phi_{z}^{\prime}-\frac{N^{2}}{g} \Phi^{\prime}\right) \hat{z}\right]+\frac{1}{2}\left(\Phi^{\prime}\right)^{2} \hat{z} \times \nabla_{h} \tilde{q}\right\} .
\end{aligned}
$$

The flux $J$ obeys the group velocity property in the WKB limit.

The pseudomomentum properties of the system are described by Shepherd (1989).

\section{REFERENCES}

ARNOL'D, V. I. 1966 On an a priori estimate in the theory of hydrodynamical stability. Izv. Vyssh. Uchebn. Zaved. Matematika 54 (5), 3-5. (English transl. Am. Math. Soc. Transl., Series 2, 79, 267-269 (1969).)

BLUMEN, W. 1978 A note on horizontal boundary conditions and stability of quasi-geostrophic flow. J. Atmos. Sci. 35, 1314-1318.

Bretherton, F. P. \& Garrett, C. J. R. 1968 Wavetrains in inhomogeneous moving media. Proc. $R$. Soc. Lond. A 302, 529-554.

Cullen, M. J. P. \& PURSer, R. J. 1984 An extended Lagrangian theory of semi-geostrophic frontogenesis. J. Atmos. Sci. 41, 1477-1497.

Hoskins, B. J. 1975 The geostrophic momentum approximation and the semi-geostrophic equations. J. Atmos. Sci. 32, 233-242. 
Hoskins, B. J. 1982 The mathematical theory of frontogenesis. Ann. Rev. Fluid Mech. 14, 131-151.

Hosxins, B. J., McINTYre, M. E. \& RoBertson, A.W. 1985 On the use and significance of isentropic potential-vorticity maps. Q.J. R. Met. Soc. 111, 877-946.

KushneR, P. J. 1993 Nonlinear stability and wave, mean-flow interaction in semi-geostrophic theory. In Ninth Conf. on Atmospheric and Oceanic Waves and Stability. Preprint volume, pp. 363-366. American Meteorological Society.

Kushner, P. J. 1995 A generalized Charney-Stern theorem for semi-geostrophic dynamics. Tellus (In press).

KUSHNER, P. J. \& SHEPHERD, T. G. 1995 Wave-activity conservation laws and stability theorems for semi-geostrophic dynamics. Part 1. Pseudomomentum-based theory. J. Fluid Mech. 290, 67-104 (referred to herein as Part 1).

MCINTYRE, M. E. \& ShEPHERD, T. G. 1987 An exact local conservation theorem for finite-amplitude disturbances to non-parallel shear flows, with remarks on Hamiltonian structure and on Arnol'd's stability theorems. J. Fluid Mech. 181, 527-565 (referred to herein as MS87).

MagnusdottiR, G. \& SCHUBert, W. H. 1990 The generalization of semi-geostrophic theory to the $\beta$-plane. J. Atmos. Sci. 47, 1714-1720 (referred to herein as MSc90).

MAGnuSDOtTIR, G. \& Schubert, W. H. 1991 Semigeostrophic theory on the hemisphere. J. Atmos. Sci. 48, 1449-1456.

ROUlSTONE, I. \& NORBURY, J. 1994 A Hamiltonian structure with contact geometry for the semigeostrophic equations. J. Fluid Mech. 272, 211-233.

SALMON, R. 1985 New equations for nearly geostrophic flow. J. Fluid Mech. 153, 461-477.

SALMON, R. 1988 Semigeostrophic theory as a Dirac-bracket projection. J. Fluid Mech. 196, 345-358.

SCHUBERT, W. H. \& MAGNuSDOTTIR, G. 1994 Vorticity coordinates, transformed primitive equations and a canonical form for balance models. J. Atmos. Sci. 51, 3309-3319.

SHEPHERD, T. G. 1989 Nonlinear saturation of baroclinic instability. Part II: Continuously stratified fluid. J. Atmos. Sci. 46, 888-907.

SHEPHERD, T. G. 1990 Symmetries, conservation laws, and Hamiltonian structure in geophysical fluid dynamics. Adv. Geophys. 32, 287-338.

ShutTs, G. J. \& Cullen, M. J. P. 1987 Parcel stability and its relation to semi-geostrophic theory. J. Atmos. Sci. 44, 1318-1330.

Swaters, G. E. 1986 A nonlinear stability theorem for baroclinic quasigeostrophic flow. Phys. Fluids 29, 5-6.

Warn, T., Bokhove, O., Shepherd, T. G. \& Vallis, G. K. 1995 Rossby-number expansions, slaving principles, and balance dynamics. Q.J. R. Met. Soc. 121, 723-739.

WhItE, A. A. 1977 Modified quasi-geostrophic equations using geometric height as vertical coordinate. Q. J. R. Met. Soc. 103, 383-396. 